\title{
Genome-wide identification and expression analysis of the VQ gene family in soybean (Glycine max)
}

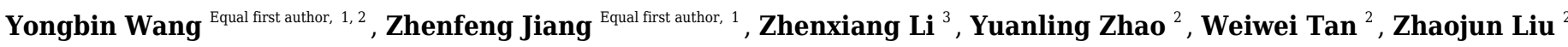 \\ , Shaobin Cui ${ }^{4}$, Xiaoguang Yu ${ }^{4}$, Jun Ma ${ }^{4}$, Guangjin Wang ${ }^{\text {Corresp., } 5}$, Wenbin Li ${ }^{\text {Corresp. } 1}$ \\ ${ }^{1}$ Key Laboratory of Soybean Biology in Chinese Ministry of Education $\square$ Key Laboratory of Soybean Biology and Breeding/Genetics of Chnese Agriculture \\ Ministry, Northeast Agricultural University, Harbin, Heilongjiang, China \\ 2 Biotechnology Research Institute, Heilongjiang Academy of Agricultural Sciences, Harbin, Heilongjiang, China \\ 3 Harbin Normal University, Harbin, Heilongjiang, China \\ 4 Heilongjiang Academy of Agricultural Sciences, Harbin, Heilongjiang, China \\ 5 Soybean Research Institute, Heilongjiang Academy of Agricultural Sciences, Harbin, Heilongjiang, China \\ Corresponding Authors: Guangjin Wang, Wenbin Li \\ Email address: gjw1962@yeah.net, wenbinli@neau.edu.cn
}

Background. VQ proteins, the plant-specific transcription factors, are involved in plant development and multiple stresses; however, only few articles systematic reported the VQ genes in soybean. Methods. In total, we identified $75 \mathrm{GmVQ}$ genes, which were classified into 7 groups (I-VII). Conserved domain analysis indicated that VQ gene family members all contain the $V Q$ domains. $V Q$ genes from the same evolutionary branches of soybean shared similar motifs and structures. Promoter analysis revealed that cis-elements related to stress responses, phytohormone responses and controlling physical as well as reproductive growth. Based on the RNA-seq and qRT-PCR analysis, GmVQ genes were showed expressing in nine tissues, suggesting their putative function in many aspects of plant growth and development as well as response to stress in Glycine max. Results. This study aims to understand the roles of $V Q$ genes in various development processes and their expression patterns in responses to stimuli. Our results provide basic information in identification and classification of GmVQ genes. Further experimental analysis will allows us to know the functions of GmVQs participation in plant growth and stress responses. 


\section{Genome-wide identification and expression analysis of the}

\section{VQ gene family in soybean (Glycine max)}

1

2

3

4

5

6

Corresponding Author:

15 Wenbin $\mathrm{Li}^{1}$ 150086, PR China China

Guangjin Wang 5

* Equal contributors.

Yongbin Wang ${ }^{1,2^{*}}$, Zhenfeng Jiang ${ }^{1 *}$, Zhenxiang $\mathrm{Li}^{3}$, Yuanling Zhao ${ }^{2}$, Weiwei Tan², Zhaojun $\mathrm{Liu}^{2}$, Shaobin Cui ${ }^{4}$, Xiaoguang $\mathrm{Yu}^{4}$, Jun $\mathrm{Ma}^{4}$, Guangjin Wang ${ }^{5}$, Wenbin $\mathrm{Li}^{1}$

${ }^{1}$ Key Laboratory of Soybean Biology in Chinese Ministry of Education, Key Laboratory of Soybean Biology and Breeding/Genetics of Chinese Agriculture Ministry, Northeast Agricultural University, Harbin, Heilongjiang,150030, PR China

${ }^{2}$ Biotechnology Research Institute Heilongjiang Academy of Agricultural Sciences, Harbin, Heilongjiang,

${ }^{3}$ Harbin Normal University, Harbin, Heilongjiang, 150025,PR China

${ }^{4}$ Heilongjiang Academy of Agricultural Sciences, Harbin, Heilongjiang, 150086,PR China

${ }^{5}$ Soybean Research Institute Heilongjiang Academy of Agricultural Sciences, Harbin, Heilongjiang, 150086,PR

NO.600 Changjiang Street,Xiangfang District,HarBin, Heilongjiang , 150030, China

Email address: wenbinli@neau.edu.cn

NO.368 Xuefu road ,Nangang District,Harbin, Heilongjiang , 150086, China

Email address: gjw1962@yeah.net 
Abstract

Background. VQ proteins, the plant-specific transcription factors, are involved in plant development and multiple stresses; however, only few articles systematic reported the $V Q$ genes in soybean.

Methods. In total, we identified $75 \mathrm{GmVQ}$ genes, which were classified into 7 groups ( I -VII). Conserved domain analysis indicated that $V Q$ gene family members all contain the $V Q$ domains. $V Q$ genes from the same evolutionary branches of soybean shared similar motifs and structures. Promoter analysis revealed that cis-elements related to stress responses, phytohormone responses and controlling physical as well as reproductive growth. Based on the RNA-seq and qRT-PCR analysis, $G m V Q$ genes were showed expressing in nine tissues, suggesting their putative function in many aspects of plant growth and development_as well as response to stress in Glycine max. Results. This study aims to understand the roles of $V Q$ genes in various development processes and their expression patterns in responses to stimuli. Our results provide basic information in identification and classification of $G m V Q$ genes. Further experimental analysis will allows us to know the functions of GmVQs participation in plant growth and stress responses.

\section{Introduction}

$V Q$ genes are plant specific genes, which involved in plant development and multiple stress responses (Cheng et al., 2012). A conserved amino acid region has been identified within them, which composed of approximately 50-60 amino acids with a highly conserved the FxxhVQxhTG motif_(Jing \& Lin, 2015). The $V Q$ domain possesses multiple biological functions in $V Q$ proteins, such as the mutant strain of $A t V Q 14$ (changes from IVQQ to EDLE) in the $V Q$ domain result in producing small seeds, nevertheless the mutations in other locations does not have this characteristic (Wang et al., 2010). Furthermore, studies have reported that $V Q$ genes are different in plants and do not have any intron in higher plants, whereas most $V Q$ genes contain one or 
48

49

50

more introns in moss (Li et al., 2014; Jiang et al., 2018; Dong et al., 2018).VQ proteins can interact with the WRKY proteins, for example, SIB1 and SIB2 are also $V Q$ proteins, they were interacted with WRKY33 by recognizing the WRKY domain in C-terminal to activating the defense of plants (Lai et al., 2011).

$V Q$ proteins were reported in dicotyledon such as Arabidopsis thaliana (Cheng et al., 2012), Vitis vinifera (Wang et al., 2015), Camellia sinensis (Guo et al., 2018), and monocotyledon such as Oryza sativa (Kim et al., 2013), Zea mays (Song et al., 2016). VQ proteins perform a variety of functions in plant development. For example, IKU1 (AT2G35230) is one of the VQ protein, it involved in regulating endosperm development and affect the seed formation during plant growth (Garcia \& Berger, 2003). Under the far-red and low intensity of white light conditions, over expression of $A t V Q 29$ can reduces the hypocotyl growth and it has higher expression in stem cells (Perruc et al., 1999). Furthermore, VQ genes regulate varying functions under abiotic and biotic stresses. AtCaMBP25 (also named AtVQ15) overexpression in transgenic plants had highly sensitive to osmotic stress in germination and early growth of seeds (Perruc et al., 1999). AtVQ9 alleviated the activity of WRKY8 under salt stress (Hu et al., 2013). The transcript levels of AtVQ23 and AtVQ16 are strongly induced by Botrytis cinerea infection and SA stress (Lai et al., 2011).

Glycine max is an important economic crop, widely cultivated in a number of countries. They are often subjected to abiotic stresses during the growth process, such as drought, high salinity, and other abiotic stresses were severely influenced on soybean production ( $L i u \& L i$, 2010). Therefore, identification of resistance genes has great significance for improving the yield and quality of soybean through molecular breeding. In this study, we identified $75 \mathrm{VQ}$ genes of the soybean genome, and analyzed their phylogenetic, evolutionary motif, structure, promoter, and expression pattern. In addition, we analyzed the $G m V Q s$ 's expression level in different multiple abiotic stresses. Our results provide a basic information on identification and classification of $G m V Q$ genes, and further experimental analysis allows us to comprehend the functions of $G m V Q s$ participate in plant growth and stress responses. 


\section{Materials \& Methods}

\section{Identification of $V Q$ genes}

The Hidden Markov Model (HMM) profiles of the $V Q$ motif PF05678 were downloaded from the Pfam database (Punta et al., 2012). HMM searched VQ motif (PF05678) from the G. max proteins database with the values (e-value) cut-off at 0.1 (Punta et al., 2012). The integrity of the $V Q$ motif was determined using the online program SMART (http://smart.embl-heidelberg.de/) with an e-value < 0.1 (Letunic et al., 2012). In addition, the three fields (length, molecular weight, and isoelectric point) of each VQ protein were predicted by the online ExPasy program (http://www.expasy.org/tools/) (Rueda et al., 2015).

\section{Phylogenetic analysis}

To investigate the phylogenetic relationship of the $V Q$ gene families among A. thaliana, $O$. sativa, and G. max, AtVQ and OsVQ proteins were downloaded from phytozomes (http://www.phytozome.org) based on the previous studies (Cheng et al., 2012, Li et al., 2014,Goodstein et al., 2012). VQ proteins were aligned using the BioEdit program. A neighborjoining (NJ) phylogenetic tree was constructed using these proteins through MEGA7.0 software (Tamura et al., 2011). Bootstrapping was performed with 1000 replications. Genes were classified according to the distance homology with $A$. thaliana and $O$. sativa genes (Cheng et al., 2012, Li et al., 2014).

\section{Sequence alignment, motif prediction and gene structure of $G m V Q$ genes}

Multiple alignments of the VQ full length proteins were conducted using Jalview software with default parameter settings. The online MEME analysis used to identify the unknown conserved motifs (http://meme.ebi.edu.au/meme/intro.html) using the following parameters: site distribution: zero or one occurrence (of a contributing motif site) per sequence, maximum number of motifs: 20, and optimum motif width $\geq 6$ and $\leq 200$ (Bailey et al., 2015). A gene 
102

103

104

105

106

107

108

109

110

111

112

113

114

115

116

117

118

119

120

121

122

123

124

125

126

127

128

structure displaying server program (http://gsds.cbi.pku.edu.cn/index.php) was used to show the structure of Glycine max $V Q$ gene.

\section{Gene duplication and collinearity analysis}

The physical locations of the GmVQ genes on the soybean chromosomes were mapped by using MG2C website (http://mg2c.iask.in/mg2c_v2.0/). The analysis of synteny among the soybean genomes was conducted locally using a method similar to the one developed for the PGDD (http://chibba.agtec.uga.edu/duplication/) (Krzywinski et al., 2009). First, BLASTP, OrthoMCL software (http://orthomcl.org/orthomcl/about.do\#release) and MCScanX software (Wang et al., 2012) were used to search for potential homologous gene pairs ( $E<1 \mathrm{e}^{-5}$, top 5 matches) across multiple genomes. Then, these homologous pairs were used as the input for the PGDD database (http://chibba.agtec.uga.edu/duplication/). Ideograms were created using Circos (Krzywinski et al., 2009).

\section{Calculating $K a$ and $K s$}

The $K a$ and $K s$ were used to assess the selection history and divergence time of gene families ( $L i$ et al., 1981). The number of synonymous $(K s)$ and nonsynonymous $(K a)$ substitutions of duplicated $V Q$ genes was computed by using the KaKs_Calculator 2.0 with the NG method $(X u$ et al., 2018). The divergence time $(T)$ was calculated using the formula $T=K s /\left(2 \times 6.1 \times 10^{-9}\right) \times$ $10^{-6}$ million years ago (MYA) (Kim et al., 2013).

\section{$V Q$ genes expression analysis of soybean}

The expression data of $V Q$ genes in different tissues, including seed, pod, SAM, stem, flower, leaf, root, root hair and nodule, is available in Phytozome V12.1 database (https://phytozome.jgi.doe.gov/pz/portal.html). The expression profile for $V Q$ genes was utilized for generating the heatmap and k-means clustering using R 3.2.2 software (Gentleman et al., 2004). 
Plant material and treatments

131 Glycine max (Williams 82) was used in this study. Seeds were planted in a 3:1 (w/w) mixture of 132 soil and sand, germinated, and irrigated with half-strength Hoagland solution once every 2 days. 133 The seedlings were grown in a night temperature of $20^{\circ} \mathrm{C}$ and day temperature of $22^{\circ} \mathrm{C}$, relative 134 humidity of $60 \%$, and a 16/8 h photoperiod (daytime: 05:00-21:00). After 4 weeks, the 135 136 germinated seedlings were treated with 20\% PEG6000 (drought), $250 \mathrm{mM} \mathrm{NaCl}$ solution (salt), 4 ${ }^{\circ} \mathrm{C}$ (cold), $100 \mu \mathrm{M}$ abscisic acid (ABA),100 $\mu \mathrm{M}$ salicylic acid (SA) solutions. Control and treated seedlings were harvested $1 \mathrm{~h}, 6 \mathrm{~h}, 12 \mathrm{~h}$, and $24 \mathrm{~h}$ after treatment. All samples were frozen in liquid nitrogen and stored at $-80{ }^{\circ} \mathrm{C}$ until use.

\section{RNA extraction and Quantitative real-time PCR (qRT-PCR)}

Total RNA was extracted from G. max using RNAiso Plus (TaKaRa, Toyoto, Japan) according to manufacturer's instructions. The cDNA synthesis was carried out with approximately $2 \mu \mathrm{g}$ RNA using PrimeScript RT reagent Kit with gDNA Eraser (TaKaRa,Toyoto, Japan). Quantitative Real-time PCR (qRT-PCR) was performed using SYBR Premix Ex Taq II (TaKaRa, Toyoto, Japan) on an ABI Prism 7000 sequence detection system (Applied Biosystems, USA) with the primers listed in Table S1. PCR amplification was performed in accordance with SYBR Premix Ex Taq (TaKaRa, Toyoto, Japan) response system. For each sample, three technical replicates were conducted to calculate the averaged $\mathrm{Ct}$ values. Relative expression was calculated by the $2^{-\Delta \Delta \mathrm{Ct}}$ method (Livak \& Schmittgen, 2001). The actin and GAPDH genes were used as internal control.

\section{Gene Ontology Enrichment}

Once the sequences were obtained ran a BLASTX search against the UNIPROT database at a 1e30 significance level. The matches were extracted and compared to the GO annotation generated against UNIPROT hits located at EBI. The GO annotation of the GmVQ genes by using WEGO 
156

157

158

159

160

161

162

163

164

165

166

167

168

169

170

171

172

173

174

175

176

177

178

179

180

181

182

2.0 website (http://wego.genomics.org.cn/).

\section{Analyzed the cis-elements of $G m V Q$ promoters}

The cis-elements of $G m V Q$ promoters were analyzed to further understand the $G m V Q$ gene family. We examined the sequences within 1500 base pairs (bp) upstream of initiation codons (ATG) for promoter analysis and searched for these sequences in the soybean genome. The ciselements in promoters were subsequently searched using the PlantCARE database (http://bioinformatics.psb.ugent.be/webtools/plantcare/html/).

\section{Gene interaction network}

Protein sequence of $G m W R K Y$ transcription factors were obtained from the genome database of soybean, also were mapped to the WRKY proteins of Arabidopsis by BLASTP tool in the TAIR database. Subsequently, the interaction between GmVQs and GmWRKYs were forecasted based on the PAIR website (http://www.cls.zju.edu.cn/pair/), their network was drawn in Cytoscape 3.6.1.

\section{Results}

\section{Identification of GmVQs}

Hidden Markov Model (HMM) of the VQ motif (PF05678) was used to search for putative VQs in soybean proteins database. A total of $75 V Q s$ were identifiedand were named from $G m V Q 1$ to GmVQ75 based on their physical locations on the chromosomes. This is different from the previous study, which $74 \mathrm{GmVQs}$ were identified before the database updated (Wang et al., 2014; Zhou et al., 2016). ExPasy predicted that these 75 VQ proteins have different physical and chemical properties whose amino acid lengths ranged from 89 aa ( $G m V Q 37)$ to 486 aa ( GmVQ18), with an average of 223 aa and most of them were less than 300 aa. The molecular weights of these $75 \mathrm{VQ}$ proteins ranged from $10.03 \mathrm{kDa}(G m V Q 37)$ to $52.79 \mathrm{kDa}(\mathrm{GmVQ18})$ and their isoelectric points ranged from 4.29 (GmVQ69) to 10.74 (GmVQ51) (Table 1). 


\section{Phylogenetic analysis and multiple alignment of the $V Q$ genes}

185 To explore the phylogenetic relationships among the $V Q$ genes of soybean, $A$. thaliana and $O$. sativa, a NJ phylogenetic tree was constructed (Fig. 1). We found that soybean and A. thaliana have a closer relationship than rice. Based on their relationship with AtVQs and OsVQs and the characteristics of GmVQs' core domain, they were divided into 7 groups, designated Group I-VII

(Figs. 1, 2). For the 75 GmVQ proteins, Group VI contains two VQ proteins; Group V has the

biggest amount, with 17 VQ proteins. Groups I , II, III, IV, VII contain 7, 15, 8, 12, 14 members respectively. At the same time, we found 5 types of $V Q$ specificity domain: FxxxVQxLTG (54/75), FxxxVQxFTG (16/75), FxxxVQxVTG (2/75), FxxxVQxLTR (1/75), FxxxVQxLTS (1/75), besides, there is also a GmVQ protein (GmVQ10) has partial domain deletion (Fig. 2). Different types of $V Q$ domains indicate that they might have different biological functions.

\section{Conserved motifs and gene structures of the $V Q$ gene family}

We predicted that the $75 \mathrm{GmVQs}$ contained 20 conserved motifs, with the motif length ranged from 11 aa to 50 aa (Fig. S1). Every GmVQ member contains 1-7 conserved motifs (Fig. 3B). All of the proteins, excepted $G m V Q 22$, show motif 1 which contains a specialty $V Q$ domain. Additionally, an unrooted phylogenetic tree was constructed with $V Q$ protein sequences, suggested that the motifs organization of $V Q$ genes were consistent with the phylogenetic tree (Fig. 3A). Group V contains motif 4, Group IV contains motif 2. We found that most groups possess more than two motifs, suggested that every group might have special functions with a highly conserved amino acid residue. Through the $V Q$ gene structures analysis, half of the group

VI has introns; genes in group V have longer coding regions, while genes of group I have shorter coding regions than other groups (Fig. 3C). Interestingly, 78.67\% (59/75) of GmVQ genes are intronless genes. It is speculated that a large number of introns might be lost in $V Q$ genes during evolution. The phylogenetic tree shows that genes from same branches have similar 
210

\section{1}

212

213

214

215

216

217

gene structures, while those from different branches have different gene structures (Fig.3A)

\section{Chromosome location and gene duplication}

We drew a chromosomal location map of GmVQ genes on each chromosome (Fig. 4). GmVQs are distributed on all soybean chromosomes, except chromosome 16, and were densely distributed on chromosome 8 and chromosome 13, containing 13 and 7 members, respectively (Fig. 4). Most of GmVQs are distributed on the two ends of chromosomes.

Segmental or tandem duplicate in many gene families are the main expanding way in plants. To better study the evolution of GmVQ genes, we further explored gene duplication events using the MCScanX software. We found that 52 pairs of genes originated from segmental duplication, and 4 pairs of genes involved in tandem duplication events (Table S2).

\section{Evolution and divergence of the $V Q$ gene family in soybean and Arabidopsis}

With the OrthoMCL software, we found 56 paralogous pairs in soybean, 37 orthologous pairs between soybean and Arabidopsis. Some $V Q$ genes have never had any homology genes. All the paralogous and orthologous pairs are listed in Table 2. At the same time, we found that two or more $G m V Q$ genes match to one $A t V Q$ gene, implying that they might promote the expansion of the $V Q$ gene family during evolution. We calculated $\mathrm{Ka} / \mathrm{Ks}$ ratios of 55 paralogous pairs in soybean (Table 3). Most $\mathrm{Ka} / \mathrm{Ks}$ ratios are $<1$, however, the $G m V Q 54 / G m V Q 63$ and GmVQ65/GmVQ36 pairs are > 1. In addition, the genetic differentiation of the 55 gene pairs occurred between 5 and 30 MYA.

\section{Expression analysis of $G m V Q$ genes among various tissues}

$67 \mathrm{GmVQ}$ genes were investigated using available RNA-seq data from nine different tissues (seed, pod, SAM, stem, flower, leaf, root, nodule, and root hair) (Fig. 5). We found that the expression levels of the $G m V Q_{s}$ varied significantly in different tissues.Most $G m V Q$ genes were found expressed in more than one detected organ. As shown in Fig. 5, genes in group A are 
236

237

238

239

240

241

242

243

244

245

246

247

248

249

250

251

252

253

254

255

256

257

258

259

260

261

262

expressed in all analyzed tissues. The expression levels of group B in pod and stem tissues are higher. Genes in group $\mathrm{C}$ have specific expression in leaf and root.

\section{Expression patterns of $G m V Q s$ under abiotic stress}

We randomly selected $25 \mathrm{GmVQ}$ genes from 7 groups, and made sure their responses to the plant hormones-, cold-, salt-, and drought-stress (Fig. 6-10). Under ABA treatment, most genes were up-regulated whole treatment period and six genes ( $\mathrm{GmVQ6/8/31/33/59/71)}$ were obviously down-regulated at some treatment time points (Fig. 6, Table S3). The expression levels of 7 genes (GmVQ2/27/40/48/53/68/74) reached the peak at the $6 \mathrm{~h}$ treatment time point and four genes $(G m V Q 9 / 21 / 31 / 71)$ reached the lowest expression levels at the early treatment time points (0-1 $\mathrm{h}$ treatment). With SA treatment, the expression levels of most GmVQs were downregulated throughout, while $G m V Q 7$ was up-regulated at $1 \mathrm{~h}, 6 \mathrm{~h}$ and $12 \mathrm{~h}$ treatment time points (Fig. 7, Table S4). In addition, nine $G m V Q$ genes ( $G m V Q 5 / 6 / 8 / 23 / 31 / 68 / 70 / 71 / 74)$ were downregulated under all abiotic stress.

With cold treatment, the expression levels of fourteen $G m V Q$ genes (GmVQ2/7/9/28/29/31/33/40/46/48/53/59/68/74) were up-regulated throughout (Fig. 8, Table S5), while the expression levels of three genes ( $G m V Q 27 / 64 / 65)$ were down-regulated and then up-regulated during treatment. Under salt stress, the results were similar to that with cold stress treatment, most genes were up-regulated, eight genes (GmVQ9/23/27/33/65/68/70/71) were down-regulated throughout (Fig. 9, Table S6). On the contrary, under drought (PEG) stress, most genes were down-regulated, only eight genes ( $G m V Q 2 / 6 / 7 / 8 / 21 / 29 / 33 / 48)$ were up-regulated during the treatment (Fig. 10, Table S7).

\section{Cis-elements in $G m V Q$ promoters}

We found many hormone- and stress- related promoter's cis-elements in GmVQ genes. Enhancer regions (CAAT-box) and core promoter element are around -30 bp of transcription start (TATAbox). Cis-acting regulatory element (A-box) are the common cis-acting elements in the promoter. 
263 Others cis-elements that were found in the $75 \mathrm{GmVQs}$ can be classified into three groups (Fig.

264 11). Twelve cis-elements involve in the hormone responsiveness; five $c i s$-elements are stress-

265 related elements: ARE/ GC/LTR/MBS/TC; some GmVQ genes containe plant growth and

266 development elements, such as CAT-box/circadian/GCN4/HD-Zip 1/MSA-like/ RY-element. In

267 addition, some $G m V Q$ genes containe W-box motif, which is binding site for WRKY

268 transcription factor.

269

270

\section{Gene Ontology Enrichment}

271

272

273

274

275

276

277

278

279

280

\section{1}

282

To further understand the functions of the GmVQs, we performed GO annotation and GO enrichment analyses (Figure S2 and Table S8 ). The GO terms included three categories, biological process (BP), molecular function (MF) and cellular component (CC). GO enrichment confirmed that these $G m V Q s$ were enriched in the biological process (GO:0008150), regulation of biological process (GO:0050789) and biological regulation (GO:0065007) terms of the BP category. Cellular component (GO:0005575), intracellular (GO:0005622) and cell (GO:0005623) were the most abundant functions in the $\mathrm{CC}$ category (Table S8). MF was enriched in molecular function (GO:0003674) and binding (GO:0005488). The GO ebrichment suggested that GmVQs were play curcial roles in regulated of biological process.

\section{Gene interaction network analysis}

Based on the PAIR tool, we found the functions and their interactions of the GmVQs and GmWRKYs. As shown in Fig. 12A, 3 GmWRKYs are supposed to interact with GmVQ proteins, included GmWRKY115, GmWRKY149 and GmWRKY156, all of them belong to WRKY's II c group. In the Fig. 12B, we found that GmWRKYs and AtWRKYs are quite similar in their core domains, indicated that they might have same functions, such as interacted with VQ proteins.

\section{Discussion}

VQ protein is a kind of specific protein that widely exists in plant, involved in plant growth and 
290

291

292

293

294

295

296

297

298

299

300

301

302

303

304

305

306

307

308

309

310

311

312

313

314

315

316

can response to different stresses (Petersen et al., 2010; Fiil \& Petersen, 2011; Xie et al., 2010). Hence, we completed genome-wide analysis of soybean VQ proteins by bioinformatic analysis and qRT-PCR to understand their regulation when environmental changed. In the previous study, 74 GmVQ genes were identified (Wang et al., 2014; Zhou et al., 2016). After the database was updated, we identified and isolated $75 \mathrm{GmVQ}$ genes in the soybean genome. Compared with previous study, the number of genes in chromosome 2, 4 and 17 show a big difference. Soybean contains more VQ genes than that of A. thaliana (34) (Cheng et al., 2012), Populus trichocarpa (51) (Chu et al., 2016) and O. sativa (42) (Kim et al., 2013). The reason is the whole genome duplication events (WGD). There are two rounds of genome duplication, occurred at around 59 and 13 million years ago, which caused 75\% soybean genes duplicated (Jeremy et al., 2010).

$75 V Q$ genes were identified in Glycine max's genome, divided into seven groups based on their comprehensive phylogenetic tree among G. max, A. thaliana, and O. sativa. These proteins are in the shorter branches and with closer spacing, suggesting that they were highly conserved during the evolution. The more closer related genes within the same group shared more similar gene structures, either in their intron or in the exon patterns. Whereas, the variation in different groups suggested the functional diversity of the $V Q$ genes (Jiang et al., 2018). In addition, most $G m V Q$ genes $(59 ; 78.67 \%)$ were found intronless, and most $G m V Q$ genes $(64 ; 85.33 \%)$ encoded relatively small proteins with protein length less than 300 amino acid. This suggests that $V Q$ gene families were intronless and they were highly conserved during evolution. At the same time, gene duplication can help plants to adapt to different environments during their development and growth (Huang et al., 2016; Storz, 2009). The main expansion of GmVQ gene family is segmental duplication $(52 ; 92.9 \%)$, only 4 pairs of genes involved in tandem duplication events $(4 ; 7.1 \%)$. A similar phenomenon was reported in the $B r V Q$ gene family, which contains a high proportion of segmental duplication (71.9\%) and low proportion of tandem duplication (28.1\%) (Zhang et al., 2015).

Nonfunctionalization, subfunctionalization, and neofunctionalization generally take place after genome duplication, resulting in lose or fix of genes (He \& Zhang, 2005; Sandve et al., 
317 2018; Stark et al., 2017). Soybean has undergone the WGD and the whole genome triplication

318 (WGT) compared to grapevine (Wang et al., 2017). As there are 18 VQ genes in grapevine

319 genome, the predicted number of VQ genes in soybean should be more than 100 (Wang et al.,

320

321

322

323

324

325

326

327

328

329

330

331

332

333

334

335

336

337

338

339

340

341

342

343

2015). However, in this study, we only found $75 V Q$ genes in the soybean genome, suggesting that there were gene loss events after genome duplication. In addition, the Ks value of each paralogous pairs was calculated to find gene duplication events, the most duplication events in GmVQ gene occurred between 5 and 30 MYA, consistent with the recent WGD in soybean (Wang et al., 2017; Schmutz et al., 2010). The $K a / K s$ ratios in different gene pairs are different, but most gene pairs' $\mathrm{Ka} / \mathrm{Ks}$ ratios are less than one and only two gene pairs' (GmVQ54-GmVQ63 and $G m V Q 65-G m V Q 36$ ) ratios are larger than 1, implying these gene pairs undergo different selection pressure. The above analysis indicated that purifying selection played a crucial role during the evolution, conserved $V Q$ proteins evolved much slowly at the protein level.

Expression patterns of $67 \mathrm{Gm} V Q$ genes were performed to determine their tissue expression using RNA-seq data. The results showed that 24 genes were relatively highly expressed in nine tissues, indicated that they may relate to the growth and development of plants. Moreover, $76 \%$ $(57 / 75)$ and 64\% (48/75) of GmVQ genes' expression levels were obviously increased in leaves and roots, respectively. More and more studies have shown that VQ proteins played a significant role in plants development. The study of $A$. thaliana mutants showed that AtVQ8 had a certain influence on chlorophyll formation and leaf growth and development (Cheng et al., 2012). In this study, GmVQ7 and GmVQ75 were in the same evolutionary branch with AtVQ8. Their high expression in leaves indicating they might have similar function as AtVQ8. (Cheng et al., 2012). These results will help us to study the further function of soybean's VQ proteins.

Plants need to face various abiotic stresses during their growth in natural conditions, the most common of which are high salt, drought and cold (Kim et al., 2013; Wang et al., 2014; ). Except for regulation by environmental factors, $V Q$ gene family is regulated by defense-related hormones, such as SA and ABA. In our study, we selected 25 GmVQs for qRT-PCR analysis under five different stresses (salt, drought, cold, SA and ABA stresses). In this study, most 
344

345

346

347

348

349

350

351

352

353

354

355

356

357

358

359

360

361

362

363

364

365

366

367

368

369

370

$G m V Q$ genes were up-regulated with the SA treatment, the result is consistent with previous study that most AtVQ genes can response to pathogen or the SA treatment (Cheng et al., 2012). In addition, fifteen $G m V Q$ genes (e.g., $G m V Q 2 / 21 / 29 / 46$ ) were up-regulated under SA treatment, suggesting that they play a potential role in stress resitance. $56 \%$ GmVQ genes $(14 / 25)$ were upregulated, which is different with the up-regulation of $V Q$ genes in rice that only three $O s V Q$ genes were up-regulated more than two fold (Kim et al., 2013). Increasing evidence suggests that $V Q$ genes are involved in various stress response. For example, $23 \% \mathrm{ZmVQ}$ genes were upregulated, all the $V v V Q$ genes were up-regulated by drought stress (Song et al., 2016; Wang et al., 2015). Consistently, 30\% of GmVQ genes were up-regulated, GmVQ2/29/33 were highly expressed under drought stress. Nevertheless, $A t V Q^{9}$ and $A t V Q 15$ were reported can response to abiotic stress during high salinity treatment. The response of $V Q$ genes to cold stress is similar to that of Chinese cabbage (Hu et al., 2013; Zhang et al., 2015; Cheng et al., 2012). In our study, $G m V Q 5 / 6 / 7 / 31 / 46 / 58 / 59$ and $G m V Q 7 / 9 / 28$ were activated the salt and cold stresses, respectively, because that their promoter region exists in specific stress cis-elements. Besides, homologous GmVQ genes possessed similar expression pattern but may exhibit opposite expression trend under stress, such as $G m V Q^{9-G m V Q 21}$ were up-regulated under SA treatment, but $G m V Q^{9}$ was up-regulated and GmVQ29 was down-regulated during cold stress. These results suggest that $G m V Q$ genes participate in response mechanism of abiotic stresses, their regulation mechanism is complex and diverse.

As auxiliary factor, VQ genes regulate transcription, can interact with many proteins to participate in regulating complex physiological and biochemical processes of plants, such as they can interact with WRKY transcription factors (Wang et al., 2015; Lei et al., 2017; Lai et al., 2011). Studies have shown that the responses under three different pathogens, $V Q$ protein are interacted with WRKY protein in rice (Li et al., 2014). VQ proteins and WRKY proteins may form a protein complex to exercise function. We found some of the GmVQ genes interact with group I's $W R K Y$, most $V Q$ genes interact with groups I and IIc's VQ protein in various stresses in previous reports (Dong et al., 2018; Guo et al., 2018; Lei et al., 2017). The promoter analysis 
371

372

373

374

375

376

377

378

379

indicated that 23 of $75 \mathrm{GmVQ}$ genes (30.67\%) contained one or more W-box motif in their 1500 bp promoter regions, W-box were present in 78\% VvVQ genes, 91\% ZmVQ genes contained one or more W-box motif (Song et al., 2016). In the promoters of GmWRKY genes, W-boxes could regulate $G m W R K Y$ members (Dong et al., 2003). It indicates that WRKY protein affect VQ genes expression and thus responses to environmental stimuli (Dong et al., 2018; Guo et al., 2018).

\section{Conclusions}

$75 V Q$ genes were identified in the soybean genomes. All $V Q$ genes fell into seven groups ( I -

VII). $V Q$ genes from the same evolutionary branches of soybean shared similar motifs and structures. The selection pressure analysis showed that most of the paralogous pairs were under a strong purifying selection in the $G m V Q$ genes. RNA-seq analysis revealed that the $V Q$ genes had different expression patterns in different tissues, indicating that they play crucial roles in different tissue. Finally, qRT-PCR showed that the $V Q$ gene family was responsive to biotic and abiotic stresses. Our results provide a theoretical basis for further study on the function of GmVQs.

\section{Acknowledgements}

The authors would like to thank the key laboratory of crop and livestock molecular breeding of Heilongjiang Province for providing plenty of helpful manpower and material support.

\section{References}

Bailey, T.L., Johnson, J., Grant, C.E., and Noble, W.S. 2015. The MEME Suite. Nucleic Acids Research 43(W1): W39-W49 DOI 10.1093/nar/gkv416.

Cheng, Y., Zhou, Y., Yang, Y., Chi, Y.J., Zhou, J., Chen, J.Y., Wang, F., Fan, B., Shi, K., and Zhou, Y.H. 2012. Structural and functional analysis of VQ motif-containing proteins in Arabidopsis as interacting proteins of WRKY transcription factors. Plant Physiology 159(2):810-825 DOI 10.1104/pp.112.196816.

Chu, W., Liu, B., Wang, Y., Pan, F., Chen, Z., Yan, H., and Xiang, Y. 2016. Genome-wide analysis of poplar VQ gene family and expression profiling under PEG, $\mathrm{NaCl}$, and SA treatments. Tree Genetics \& Genomes 
12(6): 124 DOI 10.1007/s11295-016-1082-z.

Dong, J., Chen, C., and Chen, Z. 2003. Expression profiles of the Arabidopsis WRKY gene superfamily during plant defense response. Plant Molecular Biology 51:21-37 DOI 10.1023/A:1020780022549.

Dong, Q., Zhao, S., Duan, D., Tian, Y., Wang, Y., Mao, K., Zhou, Z., and Ma, F. 2018. Structural and functional analyses of genes encoding VQ proteins in apple. Plant Science 272:208-219 DOI 10.1016/j.plantsci.2018.04.029. Fiil, B.K., and Petersen, M. 2011. Constitutive expression of MKS1 confers susceptibility to Botrytis cinerea infection independent of PAD3 expression. Plant Signaling \& Behavior 6(10):1425-1427 DOI 10.4161/psb.6.10.16759.

Garcia, D., and Berger, F. 2003. Arabidopsis haiku mutants reveal new controls of seed size by endosperm. Plant Physiology 131(4):1661-1670 DOI 10.1104/pp.102.018762.

Gentleman, R.C., Carey, V.J., Bates, D.M., Bolstad, B., Dettling, M., Dudoit, S., Ellis, B., Gautier, L., Ge, Y., and Gentry, J. 2004. Bioconductor: open software development for computational biology and bioinformatics. Genome Biology 5:1-16 DOI 10.1186/gb-2004-5-10-r80.

Goodstein, D.M., Shu, S., Howson, R., Neupane, R., Hayes, R.D., Fazo, J., Mitros, T., Dirks, W., Hellsten, U., and Putnam, N. 2012. Phytozome: a comparative platform for green plant genomics. Nucleic Acids Research 40(D1): D1178-D1186 DOI 10.1093/nar/gkr944.

Guo, J., Chen, J., Yang, J., Yu, Y., Yang, Y., and Wang, W. 2018. Identification, characterization and expression analysis of the VQ motif-containing gene family in tea plant (Camellia sinensis). BMC Genomics 19(1): 710 DOI 10.1186/s12864-018-5107-X.

He, X., and Zhang, J. 2005. Rapid Subfunctionalization Accompanied by Prolonged and Substantial Neofunctionalization in Duplicate Gene Evolution. Genetics 169(2):1157-1164 DOI 10.1534/genetics.104.037051. Hu, Y., Chen, L., Wang, H., Zhang, L., Wang, F., and Yu, D. 2013. Arabidopsis transcription factor WRKY8 functions antagonistically with its interacting partner VQ9 to modulate salinity stress tolerance. Plant Journal 74(3):730-745 DOI 10.1111/tpj.12159.

Huang, Z., Duan, W., Song, X., Tang, J., Peng, W., Bei, Z., and Hou, X. 2016. Retention, Molecular Evolution, and Expression Divergence of the Auxin/Indole Acetic Acid and Auxin Response Factor Gene Families inBrassica RapaShed Light on Their Evolution Patterns in Plants. Genome Biology \& Evolution 8:302-316 DOI 10.1093/gbe/evv259.

Jeremy, S., Cannon, S.B., Jessica, S., Jianxin, M., Therese, M., William, N., Hyten, D.L., Qijian, S., Thelen, J.J., and Jianlin, C. 2010. Genome sequence of the palaeopolyploid soybean. Nature DOI 10.1038/nature08957. Jiang, S.Y., Sevugan, M., and Ramachandran, S. 2018. Valine-glutamine (VQ) motif coding genes are ancient and non-plant-specific with comprehensive expression regulation by various biotic and abiotic stresses. $B M C$ Genomics 19(1):342 DOI 10.1186/s12864-018-4733-7.

Jing, Y., and Lin, R. 2015. The VQ Motif-Containing Protein Family of Plant-Specific Transcriptional Regulators. Plant Physiology 169:371-378 DOI 10.1104/pp.15.00788.

Kim, D.Y., Kwon, S.I., Choi, C., Lee, H., Ahn, I., Park, S.R., Bae, S.C., Lee, S.C., and Hwang, D.J. 2013. Expression analysis of rice VQ genes in response to biotic and abiotic stresses. Gene 529(2):208-214 DOI 10.1016/j.gene.2013.08.023.

Kim, M.Y., Yang, J.K., Lee, T., and Lee, S.H. 2013. Divergence of Flowering-Related Genes in Three Legume Species. Plant Genome 6(3):841-856 DOI 10.3835/plantgenome2013.03.0008.

Krzywinski, M., Schein, J., Birol, I., Connors, J., Gascoyne, R., Horsman, D., Jones, S.J., and Marra, M.A. 
2009. Circos: an information aesthetic for comparative genomics. Genome Research 19(9):1639-1645 DOI 10.1101/gr.092759.109.

Lai, Z., Li, Y., Wang, F., Cheng, Y., Fan, B., Yu, J.Q., and Chen, Z. 2011. Arabidopsis sigma factor binding proteins are activators of the WRKY33 transcription factor in plant defense. Plant Cell 23(10):3824-3841 DOI 10.1105/tpc.111.090571.

Lei, R., Li, X., Ma, Z., Lv, Y., Hu, Y., and Yu, D. 2017. Arabidopsis WRKY2 and WRKY34 transcription factors interact with VQ20 protein to modulate pollen development and function. Plant Journal 91:962-976 DOI 10.1111/tpj.13619.

Letunic, I., Doerks, T., and Bork, P. 2012. SMART 7: recent updates to the protein domain annotation resource. Nucleic Acids Research 40(D1):302-305 DOI 10.1093/nar/gkr931.

Li, N., Li, X., Xiao, J., and Wang, S. 2014. Comprehensive analysis of VQ motif-containing gene expression in rice defense responses to three pathogens. Plant Cell Reports 33(9):1493-1505 DOI 10.1007/s00299-014-1633-4. Li, W.H., Gojobori, T., and Nei, M. 1981. Pseudogenes as a paradigm of neutral evolution. Nature 292 (5820):237-239 DOI 10.1038/292237a0.

Liu, M.M., and Li, D.M. 2010. An analysis on total factor productivity and influencing factors of soybean in China. Journal of Agricultural Science 2(2): P158 DOI 10.5539/jas.v2n2p158.

Livak, K.J., and Schmittgen, T.D. 2001. Analysis of relative gene expression data using real-time quantitative PCR and the 2(-Delta Delta C(T)) Method. Methods 25:402-408 DOI 10.1006/meth.2001.

Perruc, E., Charpenteau, M., Ramirez, B.C., Jauneau, A., Galaud, J.P., Ranjeva, R., and Ranty, B. 1999. A novel calmodulin-binding protein functions as a negative regulator of osmotic stress tolerance in Arabidopsis thaliana seedlings. Plant Journal 38:410-420 DOI 10.1111/j.1365-313X.2004.02062.x.

Petersen, K., Qiu, J.L., Lütje, J., Fiil, B.K., Hansen, S., Mundy, J., and Petersen, M. 2010. Arabidopsis MKS1 Is Involved in Basal Immunity and Requires an Intact N-terminal Domain for Proper Function. PLoS One 5(12): e14364 DOI 10.1371/journal.pone.0014364.

Punta, M., Coggill, P.C., Eberhardt, R.Y., Mistry, J., Tate, J., Boursnell, C., Pang, N., Forslund, K., Ceric, G., and Clements, J. 2012. The Pfam protein families database. Nucleic Acids Research 40(1): D290 DOI 10.1093/nar/gkh121.

Rueda, J., Realpe, T., Mejia, G.I., Zapata, E., Rozo, J.C., Ferro, B.E., and Robledo, J. 2015. Genotypic Analysis of Genes Associated with Independent Resistance and Cross-Resistance to Isoniazid and Ethionamide in Mycobacterium tuberculosis Clinical Isolates. Antimicrobial Agents \& Chemotherapy 59(12):7805-7810 DOI 10.1128/AAC.01028-15.

Sandve, S.R., Rohlfs, R.V., and Hvidsten, T.R. 2018. Subfunctionalization versus neofunctionalization after whole-genome duplication. Nature Genetics 50 DOI 10.1038/s41588-018-0162-4.

Schmutz, J., Cannon, S.B., Schlueter, J., Ma, J., Mitros, T., Nelson, W., Hyten, D.L., Song, Q., Thelen, J.J., and Cheng, J. 2010. Genome sequence of the palaeopolyploid soybean. Nature 463:178-183 DOI 10.1038/nature08957.

Song, W., Zhao, H., Zhang, X., Lei, L., and Lai, J. 2016. Genome-Wide Identification of VQ Motif-Containing Proteins and their Expression Profiles Under Abiotic Stresses in Maize. Frontiers in Plant Science 6:1177 DOI 10.3389/fpls.2015.01177.

Stark, T.L., Liberles, D.A., Holland, B.R., and O Reilly, M.M. 2017. Analysis of a mechanistic Markov model for gene duplicates evolving under subfunctionalization. BMC Evolutionary Biology 17(1):38 10.1186/s12862-016- 
482

483

484

485

486

487

0848-0.

Storz, J.F. 2009. Genome evolution: Gene duplication and the resolution of adaptive conflict. Heredity 102:99 DOI 10.1038/hdy.2008.114.

Tamura, K., Peterson, D., Peterson, N., Stecher, G., Nei, M., and Kumar, S. 2011. MEGA5: Molecular Evolutionary Genetics Analysis Using Maximum Likelihood, Evolutionary Distance, and Maximum Parsimony Methods. Molecular Biology \& Evolution 28:2731.

Wang, A.H., Garcia, D., Zhang, H.Y., Ke, F., Chaudhury, A., Berger, F., Peacock, W.J., Dennis, E.S., and Ming, L. 2010. The VQ motif protein IKU1 regulates endosperm growth and seed size in Arabidopsis. Plant Journal 63(4):670-679 DOI 10.1111/j.1365-313X.2010.04271.X.

Wang, J., Sun, P., Li, Y., Liu, Y., Yu, J., Ma, X., Sun, S., Yang, N., Xia, R., and Lei, T. 2017. Hierarchically Aligning 10 Legume Genomes Establishes a Family-Level Genomics Platform. Plant Physiology 174(1):284 DOI 10.1104/pp.16.01981.

Wang, M., Vannozzi, A., Wang, G., Zhong, Y., Corso, M., Cavallini, E., Cheng, Z.M. 2015. A comprehensive survey of the grapevine VQ gene family and its transcriptional correlation with WRKY proteins. Frontiers in Plant Science, 6:417 DOI 10.3389/fpls.2015.00417.

Wang, X., Zhang, H., Sun, G., Jin, Y., and Qiu, L. 2014. Identification of active VQ motif-containing genes and the expression patterns under low nitrogen treatment in soybean. Gene 543(2):237-243 DOI 10.1016/j.gene.2014.04.012.

Wang Y, Tang H, Debarry J D, Tan, X. , Li, J., Wang, X., Lee, T ; Jin, H. ; Marler, B. ; Guo, H. ; Kissinger, J.C. 2012. MCScanX: a toolkit for detection and evolutionary analysis of gene synteny and collinearity. Nucleic Acids Res 40(7):e49-e49 DOI 10.1093/nar/gkr1293.

Xie, Y.D., W., L.I., Guo, D., Dong, J., Zhang, Q., Y., F.U., Ren, D., Peng, M., and Xia, Y. 2010. The Arabidopsis gene SIGMA FACTOR-BINDING PROTEIN 1 plays a role in the salicylate- and jasmonate-mediated defence responses. Plant Cell \& Environment 33(5):828-839 DOI 10.1111/j.1365-3040.2009.02109.x.

Xu, L., Qiao, X., Zhang, M., and Zhang, S. 2018. Genome-Wide Analysis of Aluminum-Activated Malate Transporter Family Genes in Six Rosaceae Species, and Expression Analysis and Functional Characterization on Malate Accumulation in Chinese White Pear. Plant Science 24(3):431-442 DOI 10.1111/1744-7917.12336.

Zhang, G., Wang, F., Li, J., Qian, D., Zhang, Y., Li, H., Zhang, J., and Gao, J. 2015. Genome-Wide Identification and Analysis of the VQ Motif-Containing Protein Family in Chinese Cabbage (Brassica rapa L. ssp. Pekinensis). International Journal of Molecular Sciences 16(12):28683-28704 DOI 10.3390/ijms161226127.

Zhou Y, Yang Y, Zhou X, Chi Y, Fan B, Chen Z. 2016. Structural and Functional Characterization of the VQ Protein Family and VQ Protein Variants from Soybean. Science Reports. 6: 34663 DOI 10.1038/srep34663. 


\section{Figure legends}

516 Fig. 1 Phylogenetic tree analysis of the VQ genes in Glycine max, Arabidopsis thaliana and Oryza sativa. The 517 phylogenetic tree was constructed using MEGA 7.0 by the neighbor-joining method. The Bootstrap value was 5181,000 replicates. The three plant-specific clusters were designated as group I - VII and indicated in a specific 519 color.

Fig. 2 Multiple sequence alignment, gene structure and multiple motifs of soybean. Alignment of $V Q$ domain of 75 VQ proteins in soybean. Amino acids that are conserved throughout are shaded in different colors. The genes in different groups are in different colors.

Fig. 3 Phylogenetic tree, conserved motifs and gene structure in GmVQs. (A)Phylogenetic relationships (B) Conserved motifs of the GmVQs. Each motif is represented by a number in colored box. (C)Exon/intron structures of $G m V Q$ genes.

Fig. 4 Chromosome location and duplication events analysis in Glycine max.

530

Fig. 5 Expression analysis of $G m V Q$ genes in different tissues and different stages. The clusters were designated as group A-C. Different colors in map represent gene transcript abundance values as shown in bar at top of figure.

534

Fig. 6 qRT-PCR analysis reveals $G m V Q$ genes under ABA treatment compared to the controls. Stress treatments and time course are described in "Materials and methods". Asterisks on top of the bars indicating statistically significant differences between the stress and counterpart controls $\left({ }^{*} \mathrm{p}<0.05,{ }^{*} \mathrm{p}<0.01\right)$.

Fig. 7 qRT-PCR analysis reveals $G m V Q$ genes under SA treatment compared to the controls. Stress treatments and time course are described in "Materials and methods". Asterisks on top of the bars indicating statistically significant differences between the stress and counterpart controls $\left({ }^{*} \mathrm{p}<0.05, * * \mathrm{p}<0.01\right)$. 
543 Fig. 8 qRT-PCR analysis reveals $G m V Q$ genes under cold treatment compared to the controls. Stress

544 treatments and time course are described in "Materials and methods". Asterisks on top of the bars indicating

545 statistically significant differences between the stress and counterpart controls $\left({ }^{*} \mathrm{p}<0.05,{ }^{*} \mathrm{p}<0.01\right)$.

546

547 Fig. 9 qRT-PCR analysis reveals $G m V Q$ genes under $\mathrm{NaCl}$ treatment compared to the controls. Stress

548 treatments and time course are described in "Materials and methods". Asterisks on top of the bars indicating

549 statistically significant differences between the stress and counterpart controls $\left({ }^{*} \mathrm{p}<0.05,{ }^{*} \mathrm{p}<0.01\right)$.

550

551

552

Fig. 10 qRT-PCR analysis reveals $G m V Q$ genes under drought treatment compared to the controls. Stress treatments and time course are described in "Materials and methods". Asterisks on top of the bars indicating statistically significant differences between the stress and counterpart controls $\left({ }^{*} \mathrm{p}<0.05,{ }^{*} \mathrm{p}<0.01\right)$.

Fig.11 Number of each cis-acting element in the promoter region (1.5 kb upstream of the translation start site) of $G m V Q$ genes.

Fig.12 Interaction of GmVQ proteins with GmWRKY proteins. (A) The prediction of interaction between GmVQ proteins and GmWRKY proteins by the PAIR website, and the interaction network was draw in Cytoscape 3.6.1. (B) Sequence analysis of the WRKY domains of GmWRKY proteins and AtWRKY proteins.

\section{Table legends}

Table 1 List of all GmVQ genes information identified in the Glycine max genome.

Table 2 List of paralogous and orthologous pairs between soybean and Arabidopsis thaliana.

Table 3 List of Ka, Ks and $\mathrm{Ka} / \mathrm{Ks}$ values calculated for paralogous $V Q$ gene pairs. 


\section{Supplemental Data legends}

571 Supplemental Fig. 1 Sequence logo of motifs in $G m V Q$ genes.

572

573 Supplemental Fig. 2 Gene ontology categories assigned of the GmVQ genes

574

Supplemental Table 1 List of primers used in qRT-PCR.

576

Supplemental Table 2 List of VQ gene duplication events

Supplemental Table 3 Raw data for the ABA stress.

580

Supplemental Table 4 Raw data for the SA stress.

582

583 Supplemental Table 5 Raw data for the salt stress.

Supplemental Table 6 Raw data for the drought stress.

587 Supplemental Table 7 Raw data for the cold stress.

Supplemental Table $8 \mathrm{GO}$ terms of the GmVQ genes 
Figure 1

Phylogenetic tree analysis of the VQ genes in Glycine max, Arabidopsis thaliana and Oryza sativa.

The phylogenetic tree was constructed using MEGA 7.0 by the neighbor-joining method. The Bootstrap value was 1,000 replicates. The three plant-specific clusters were designated as group I-VII and indicated in a specific color. 


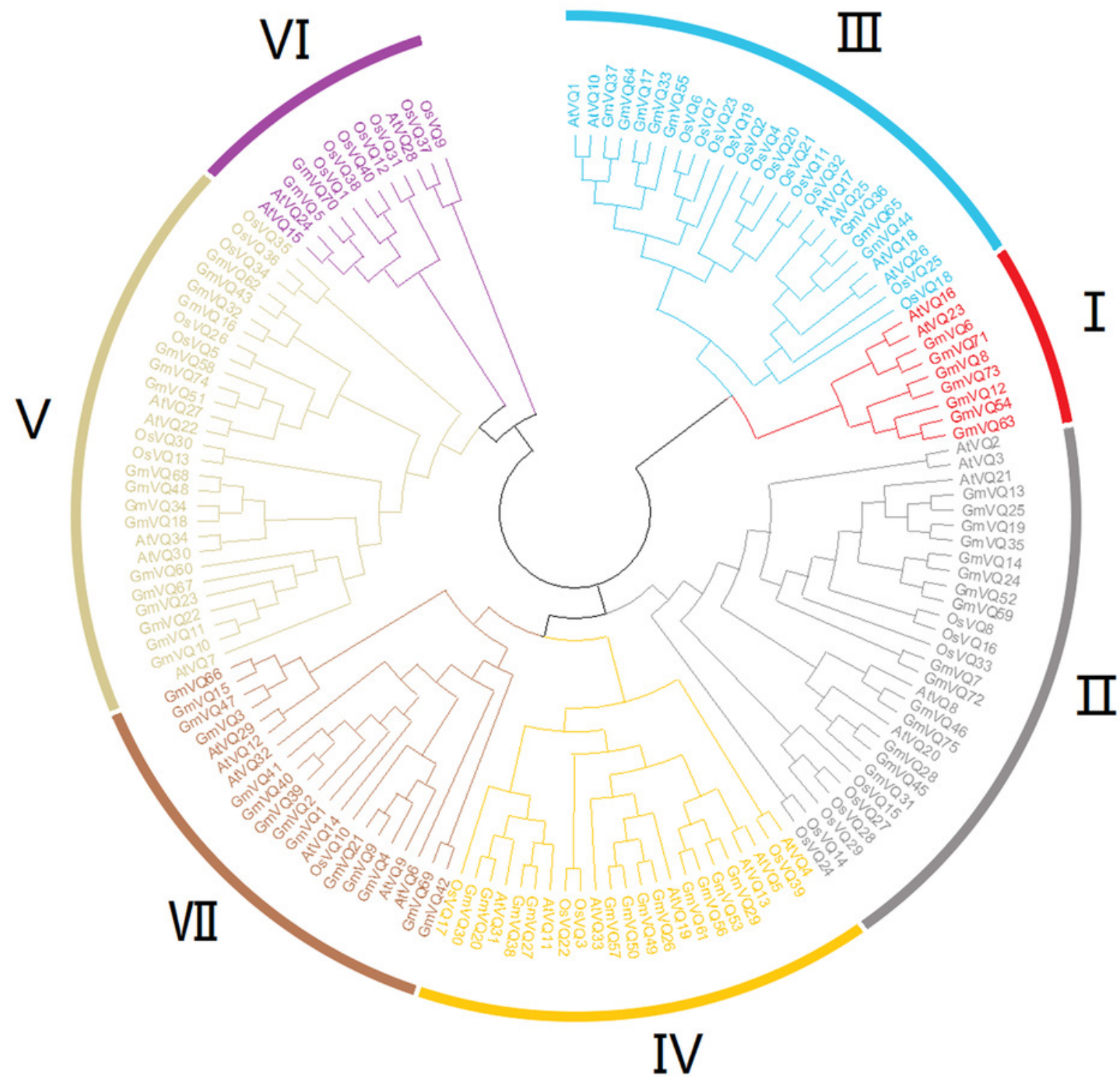


Figure 2

Multiple sequence alignment, gene structure and multiple motifs of soybean.

Alignment of VQ domain of 75 VQ proteins in soybean. Amino acids that are conserved throughout are shaded in different colors. The genes in different groups are in different colors.

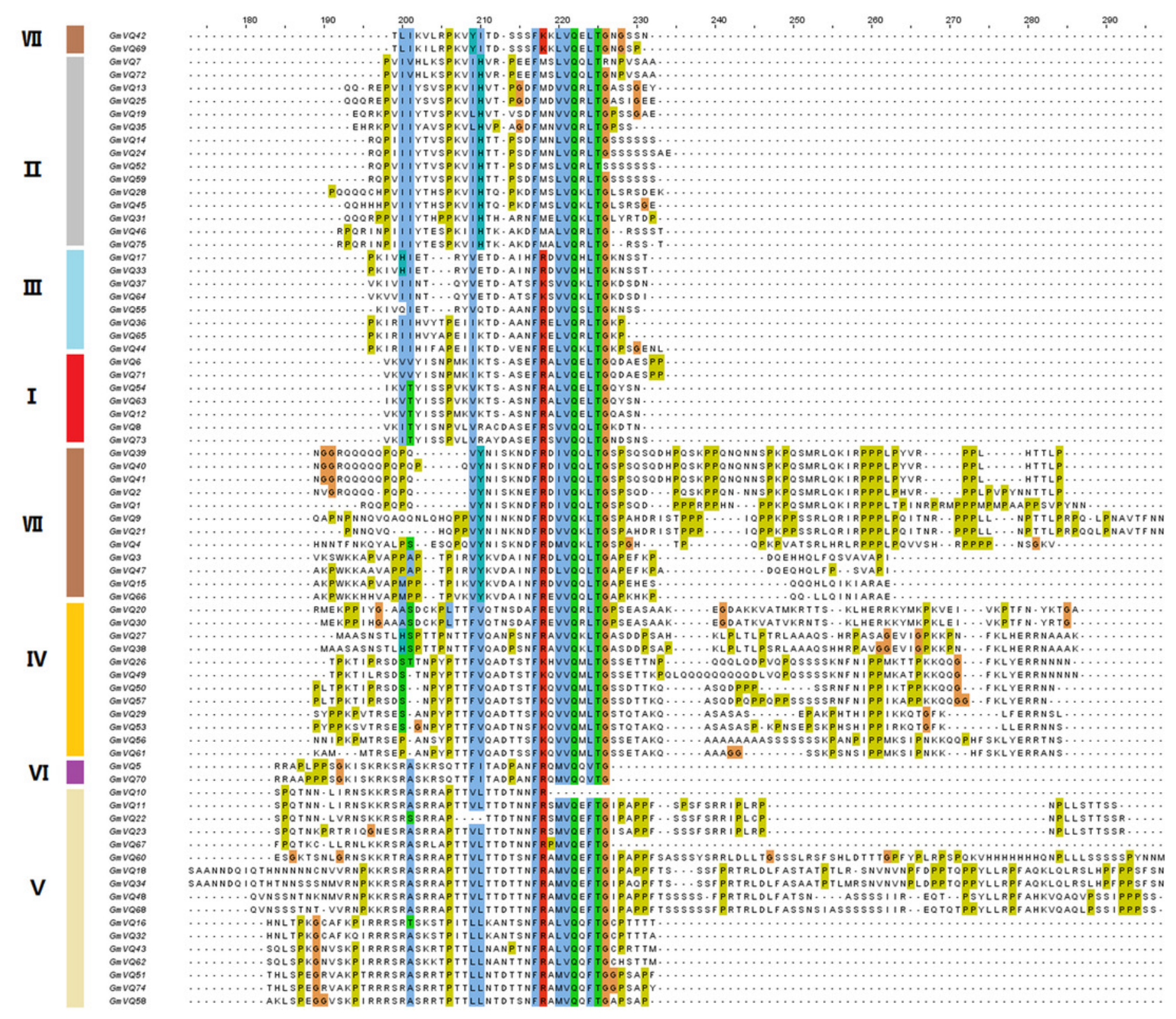


Figure 3

Phylogenetic tree, conserved motifs and gene structure in GmVQs.

(A) Phylogenetic relationships (B) Conserved motifs of the GmVQs. Each motif is represented by a number in colored box. (C) Exon/ intron structures of GmVQ genes.
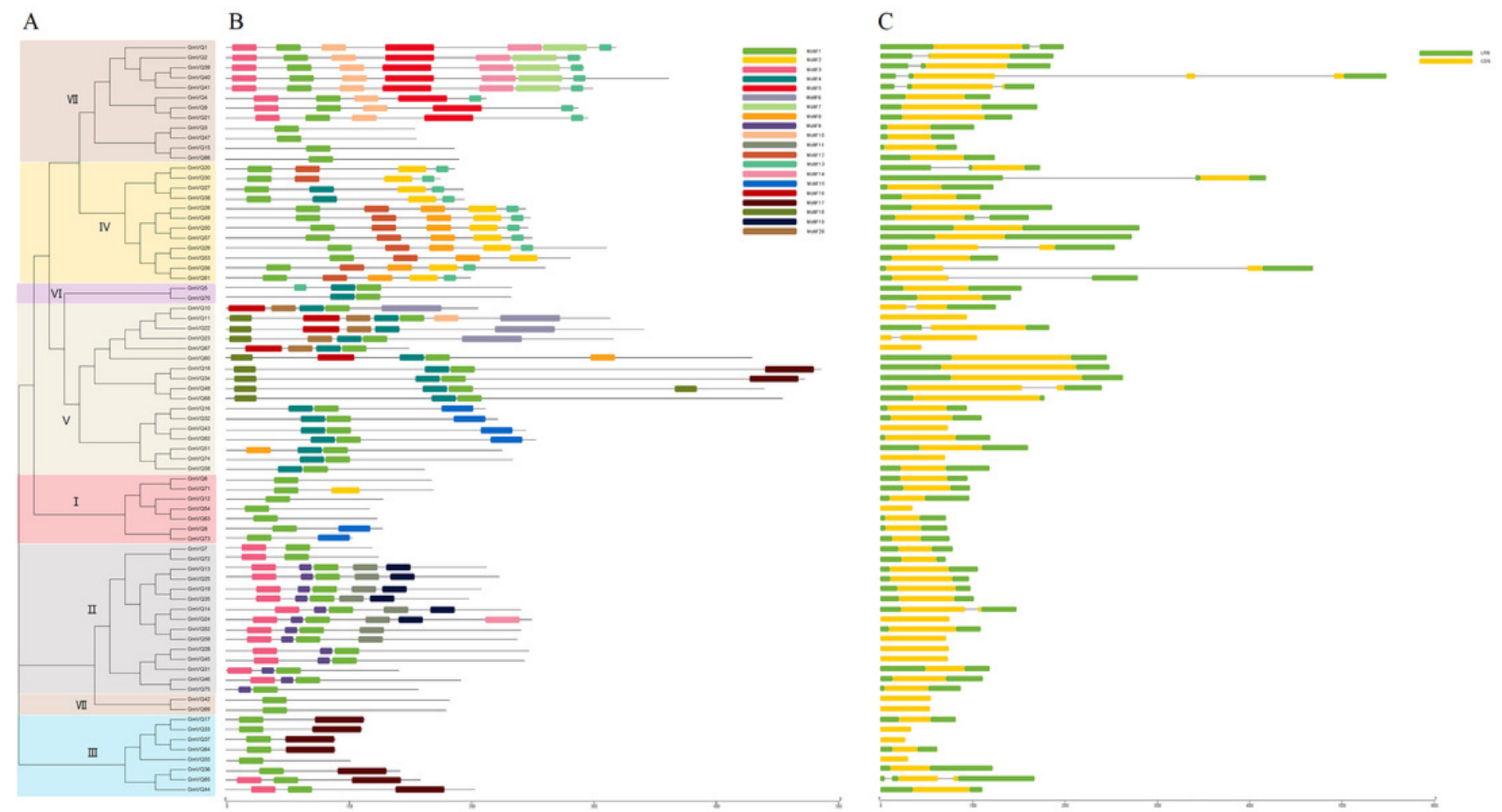
Figure 4

Chromosome location and duplication events analysis in Glycine max. 

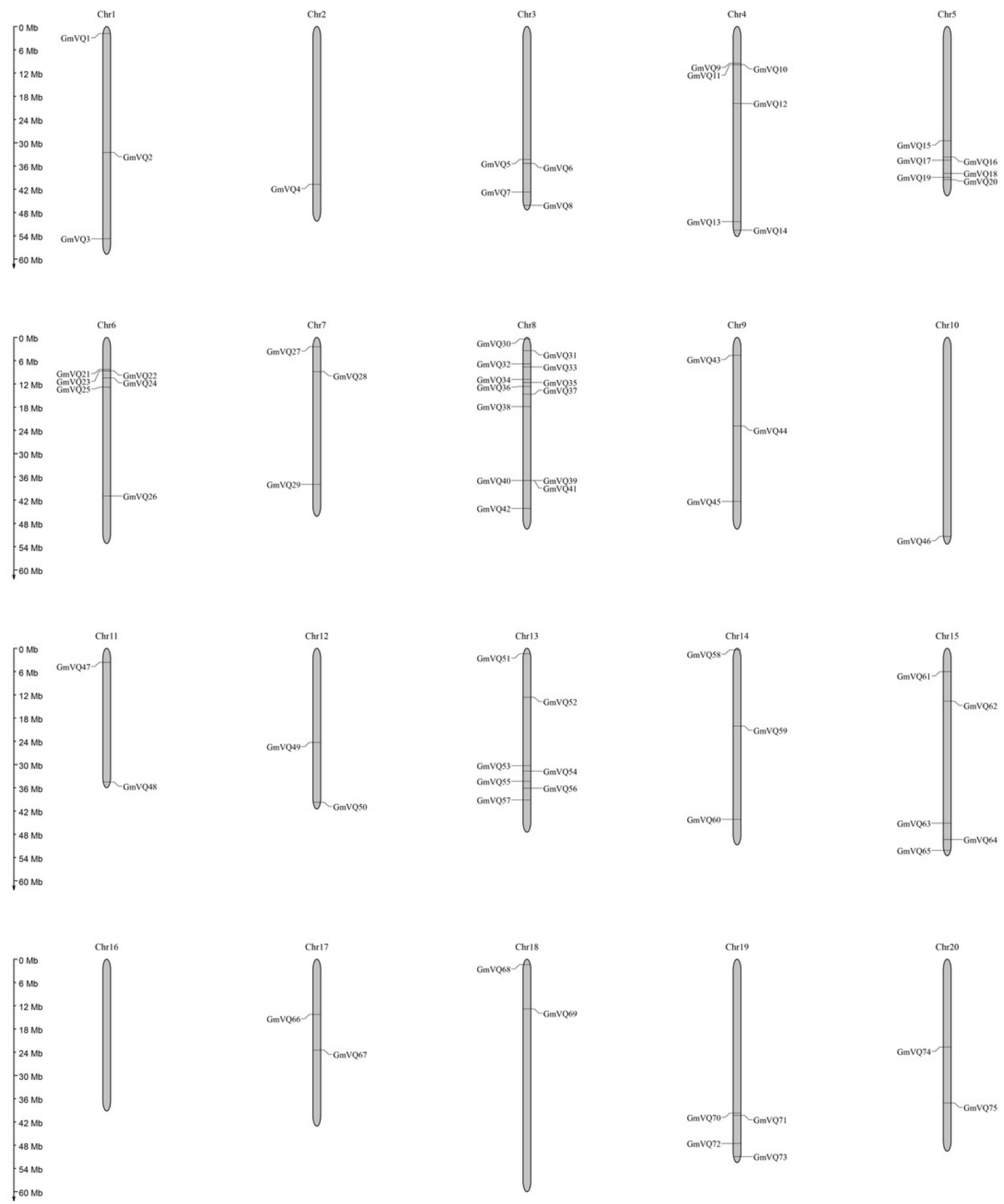
Figure 5

Expression analysis of GmVQ genes in different tissues and different stages.

The clusters were designated as group A-C. Different colors in map represent gene transcript abundance values as shown in bar at top of figure.

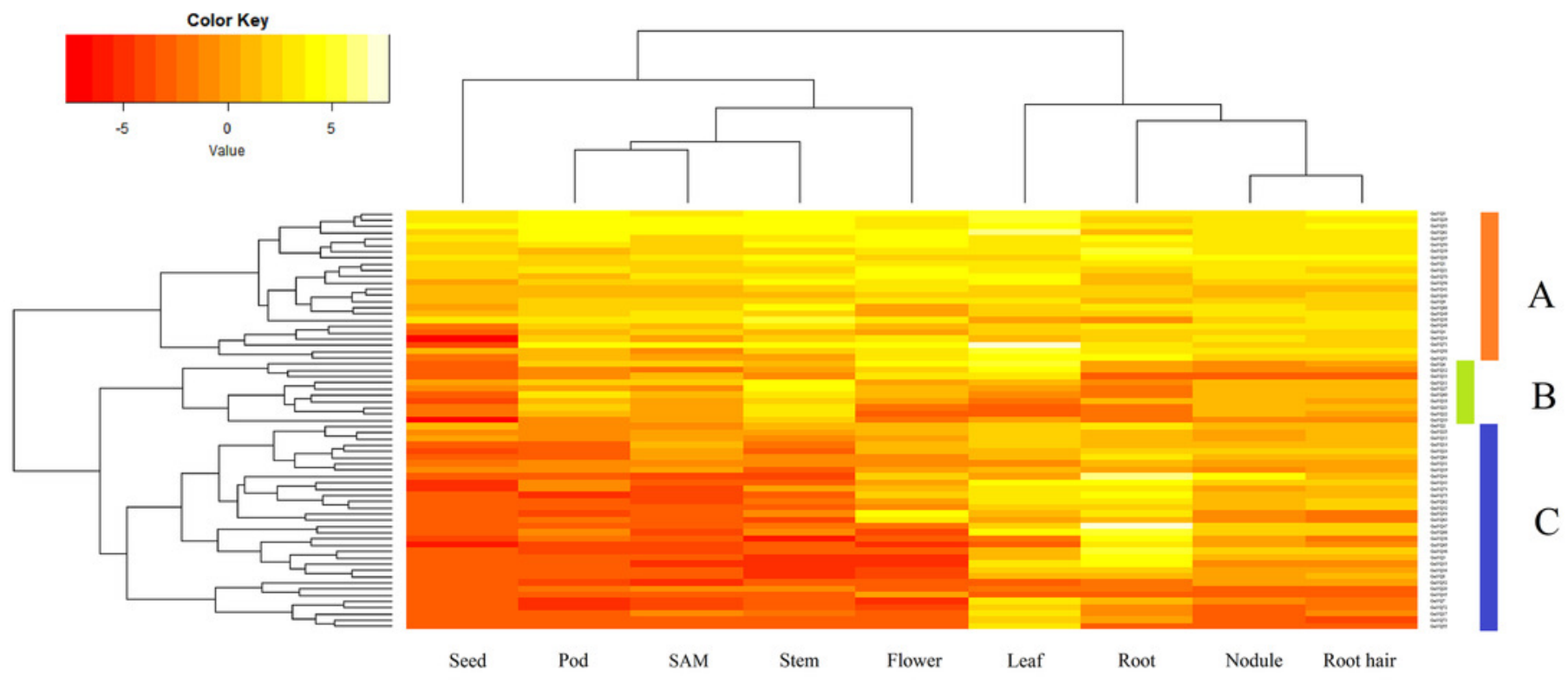


Figure 6

qRT-PCR analysis reveals GmVQ genes under ABA treatment compared to the controls.

Stress treatments and time course are described in "Materials and methods". Asterisks on top of the bars indicating statistically significant differences between the stress and counterpart controls $\left(* p<0.05,{ }^{* *} p<0.01\right)$.

\section{ABA}
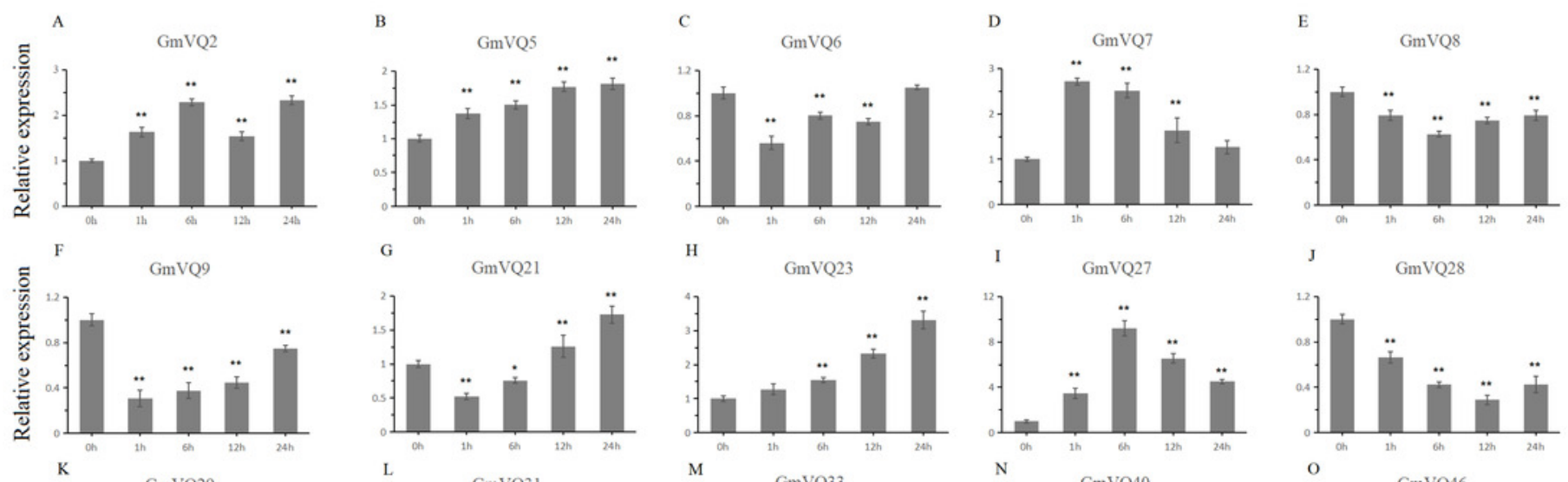

$\mathrm{H}$

GmVQ23

GmVQ27

GmVQ28
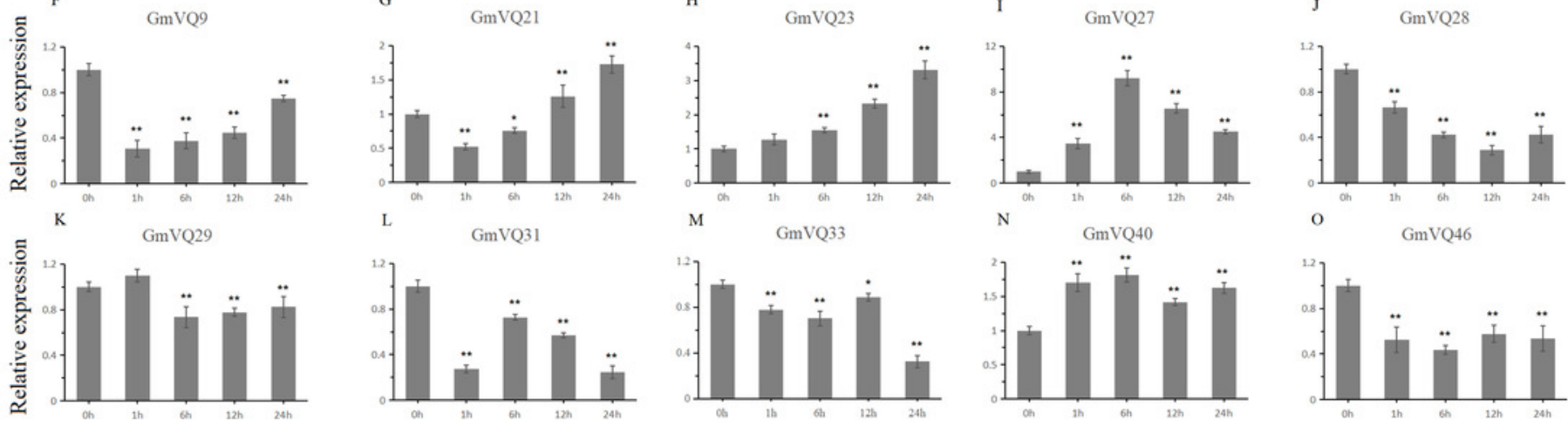

GmVQ31

GmVQ33

GmVQ46
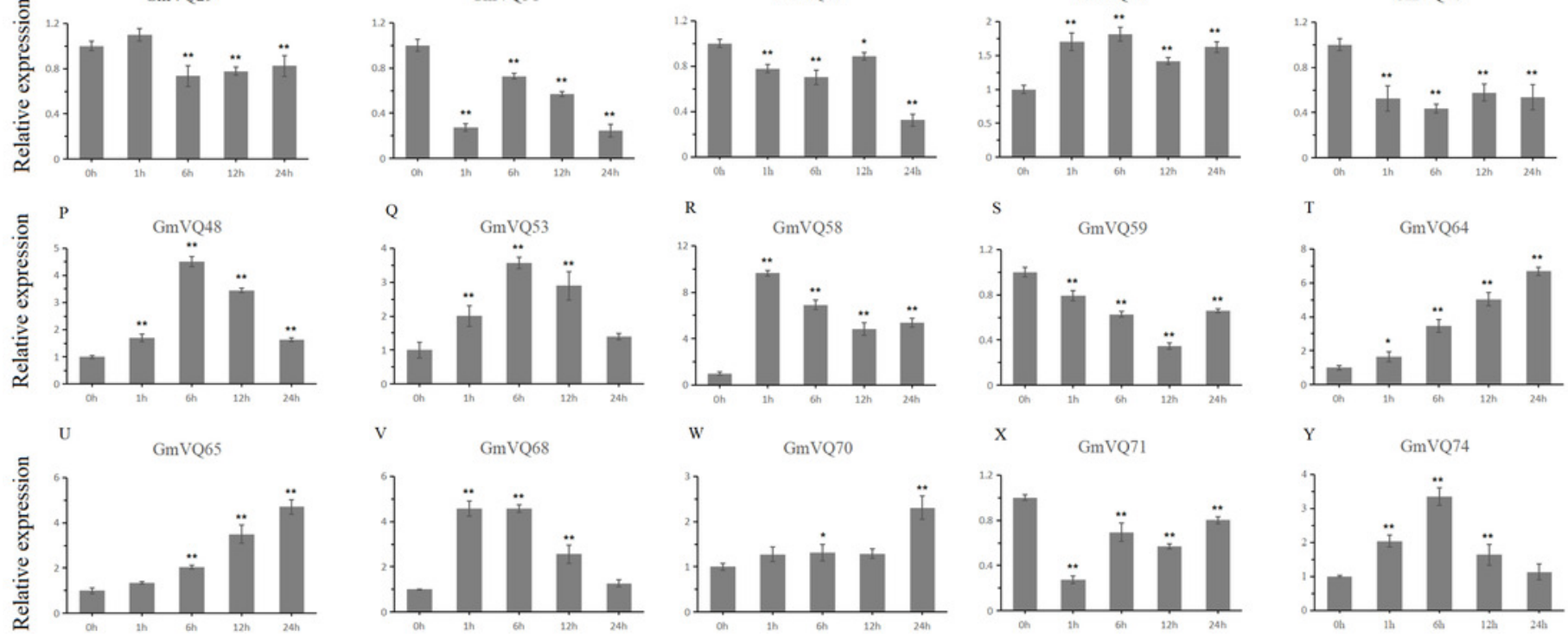
Figure 7

qRT-PCR analysis reveals GmVQ genes under SA treatment compared to the controls.

Stress treatments and time course are described in "Materials and methods". Asterisks on top of the bars indicating statistically significant differences between the stress and counterpart controls $\left(* p<0.05,{ }^{* *} p<0.01\right)$.
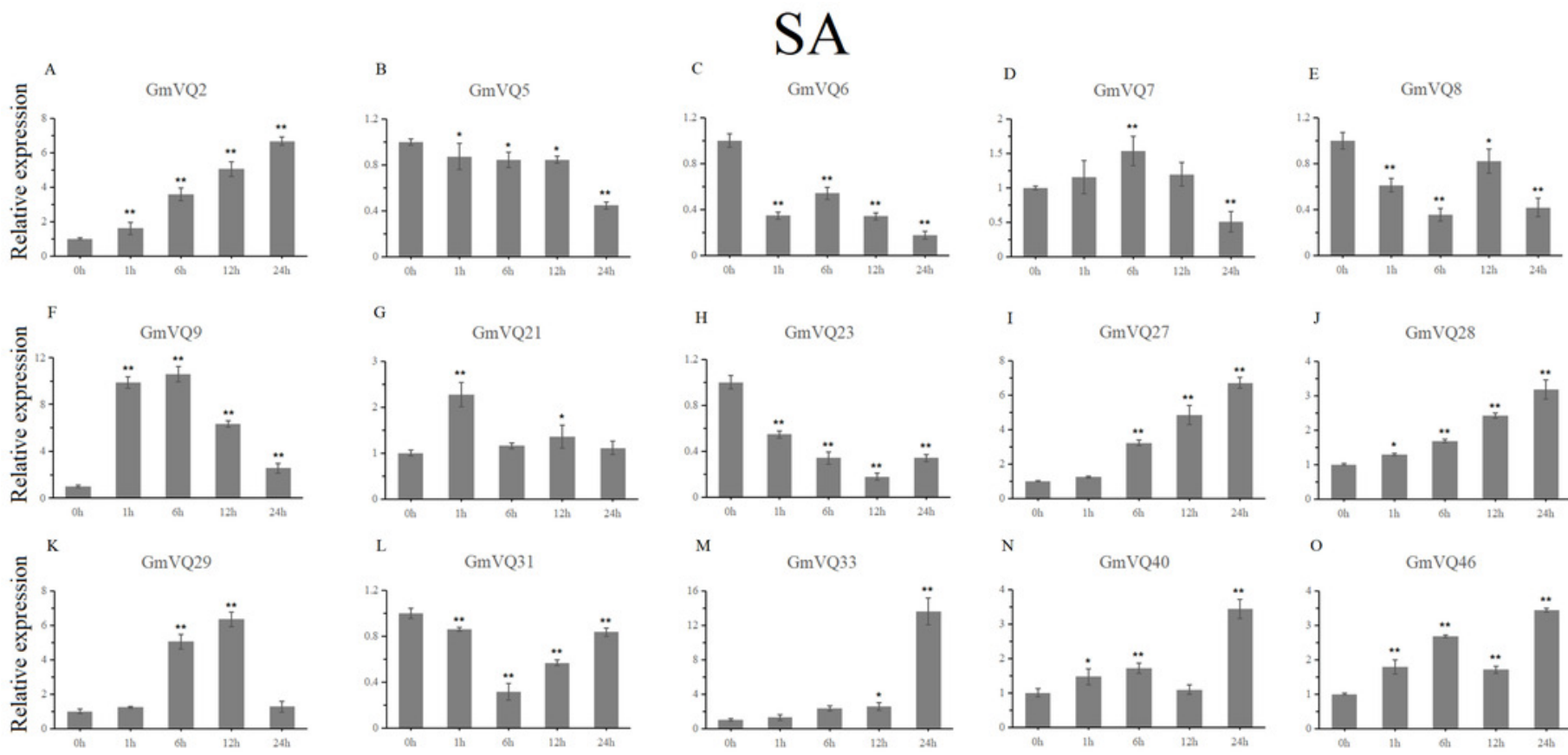

M

$\mathrm{N}$

GmVQ40
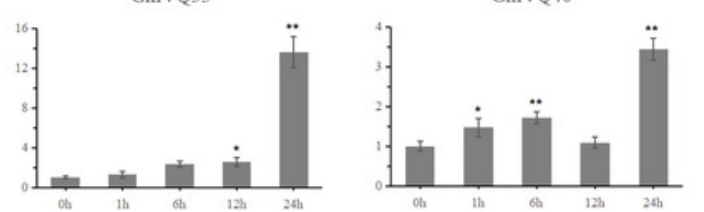

o

GmVQ46
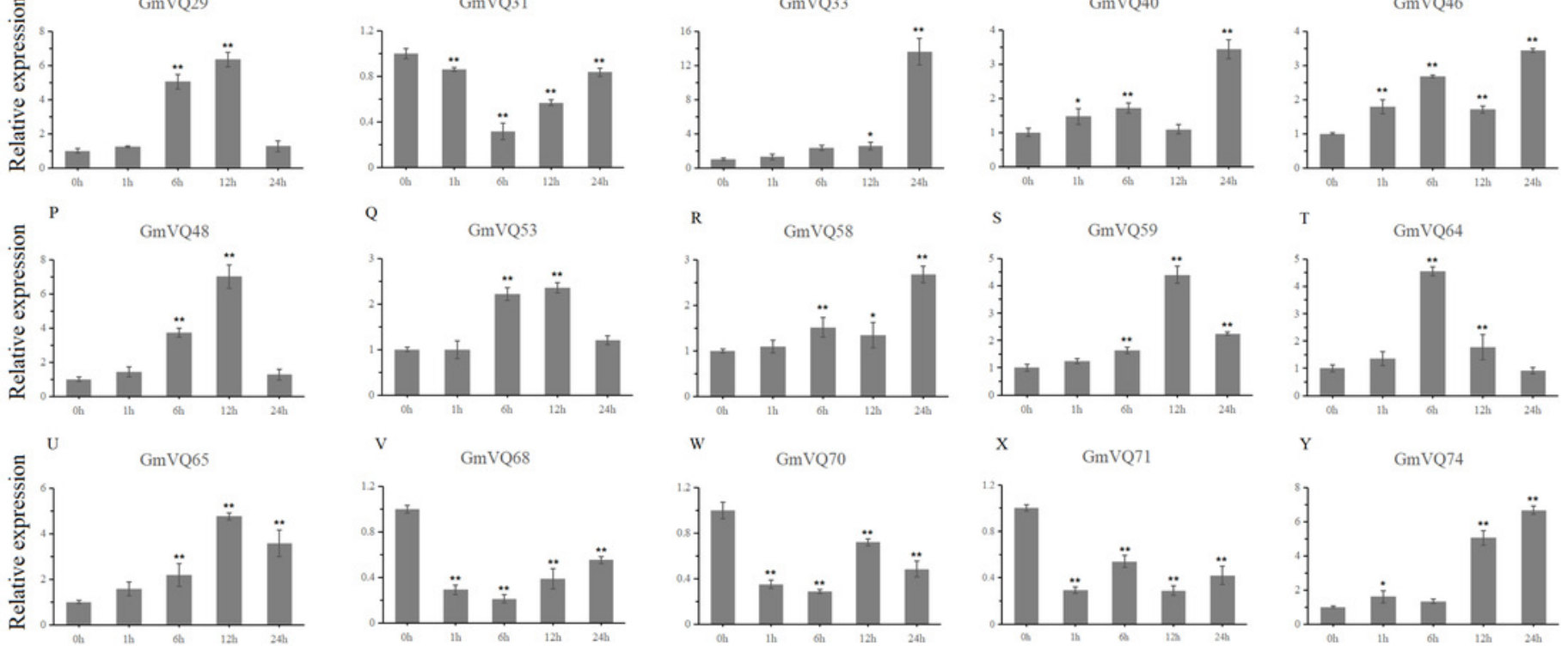
Figure 8

qRT-PCR analysis reveals GmVQ genes under cold treatment compared to the controls.

Stress treatments and time course are described in "Materials and methods". Asterisks on top of the bars indicating statistically significant differences between the stress and counterpart controls $\left(* p<0.05,{ }^{* *} p<0.01\right)$.

Cold
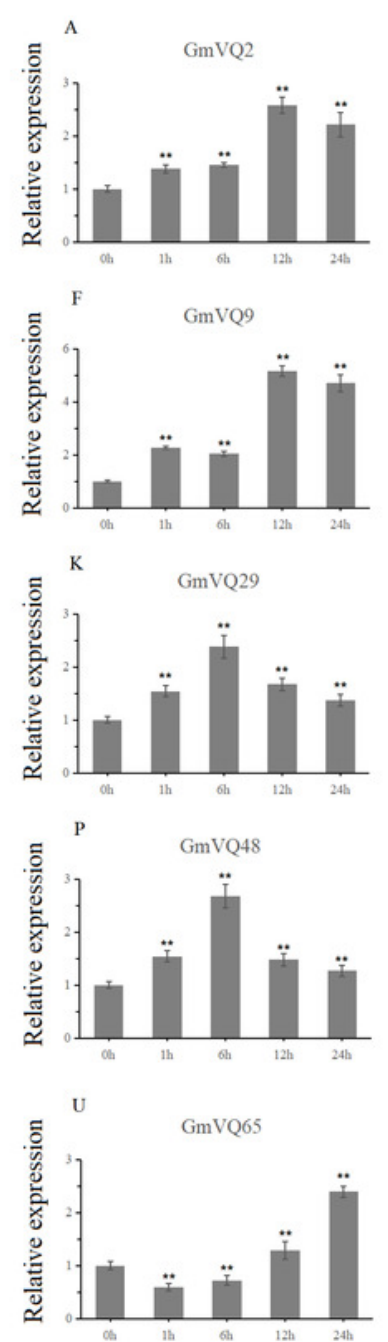

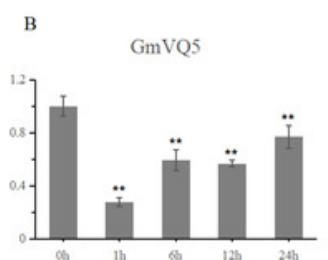

GmVQ21

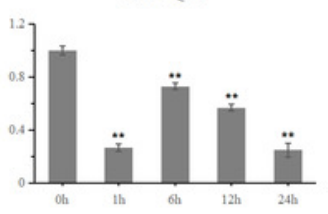

GmVQ31

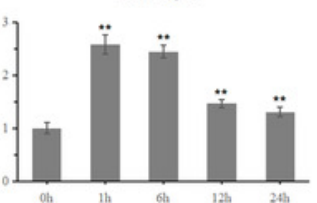

Q GmVQ53
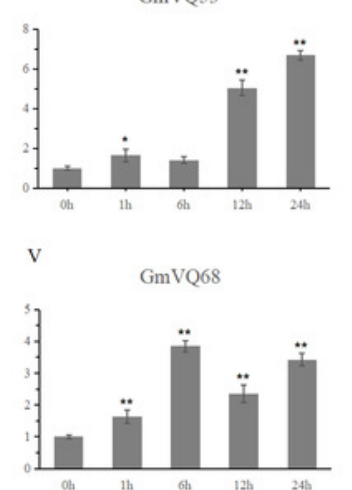

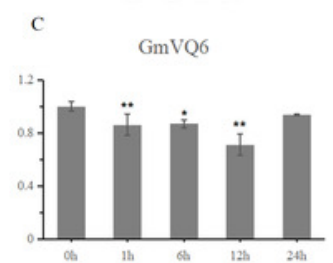

GmVQ23
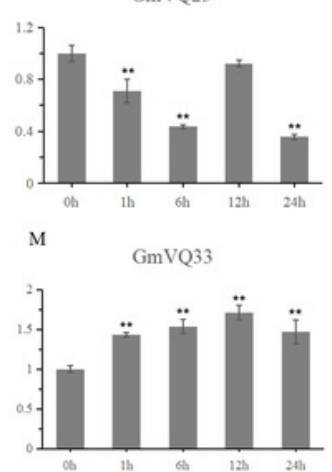

GmVQ58

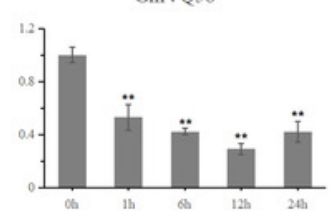

W

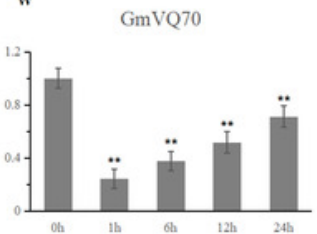

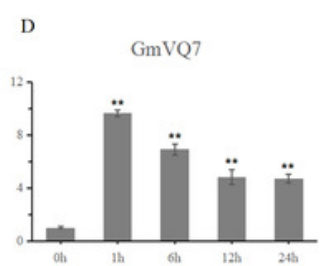
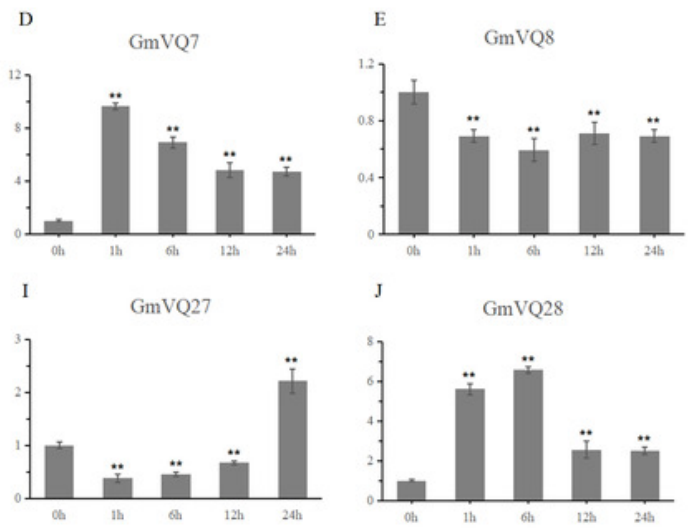

N

GmVQ40

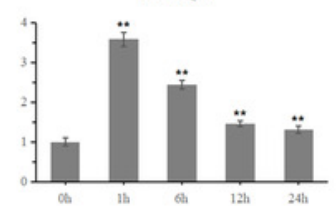

GmVQ59

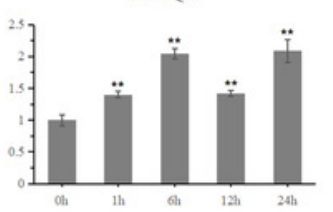

GmVQ71

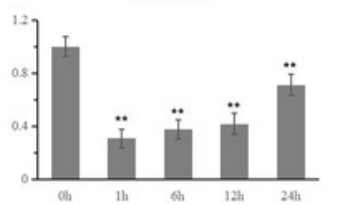

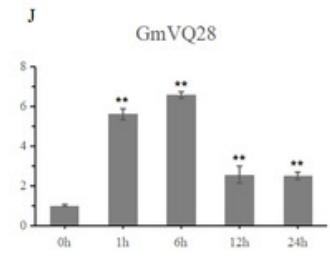

O GmVQ46

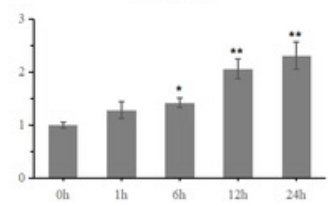

GmVQ64

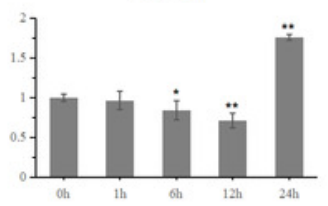

GmVQ74

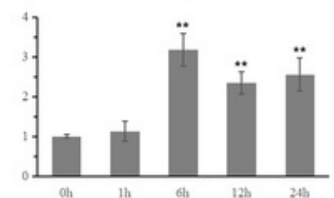


Figure 9

qRT-PCR analysis reveals GmVQ genes under $\mathrm{NaCl}$ treatment compared to the controls.

Stress treatments and time course are described in "Materials and methods". Asterisks on top of the bars indicating statistically significant differences between the stress and counterpart controls $\left(* p<0.05,{ }^{* *} p<0.01\right)$.
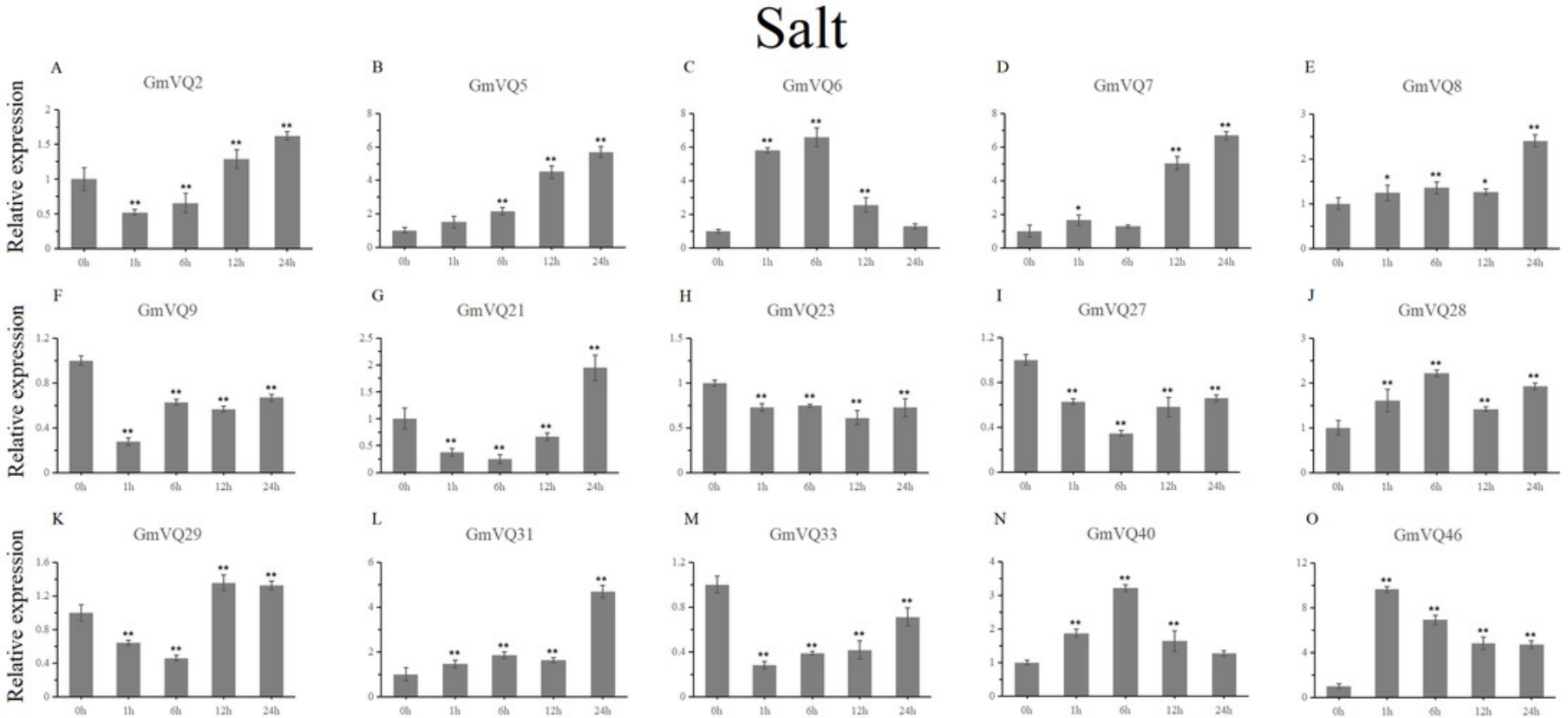

M

GmVQ33

GmVQ40

GmVQ46
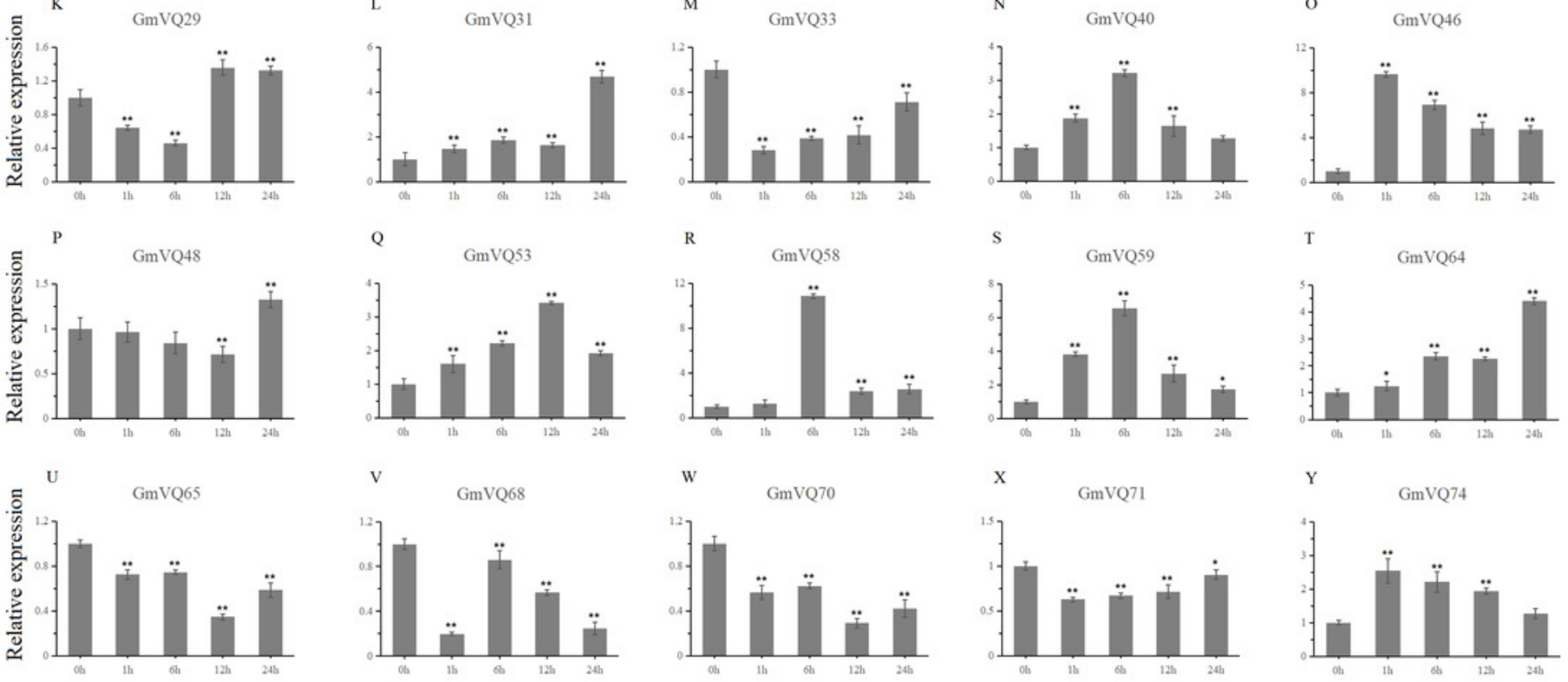
Figure 10

qRT-PCR analysis reveals GmVQ genes under drought treatment compared to the controls.

Stress treatments and time course are described in "Materials and methods". Asterisks on top of the bars indicating statistically significant differences between the stress and counterpart controls $(* p<0.05, * * p<0.01)$.
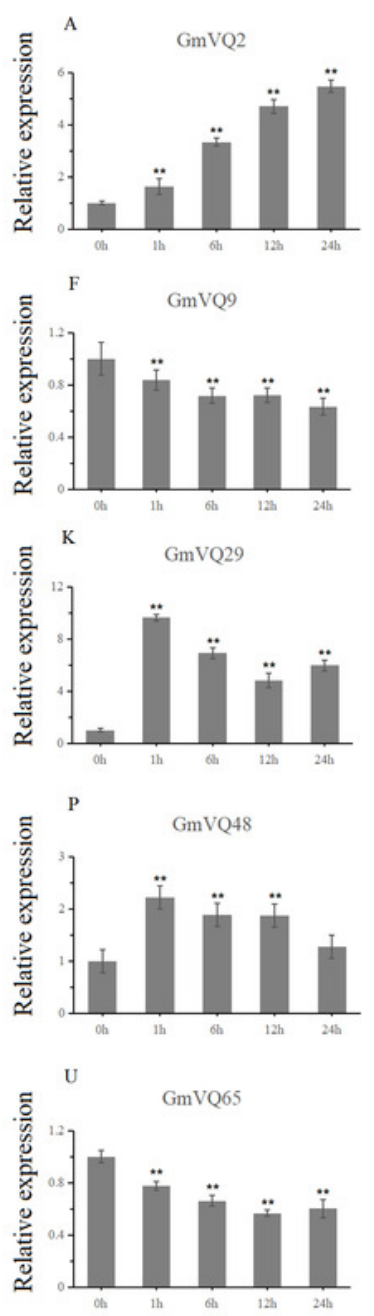

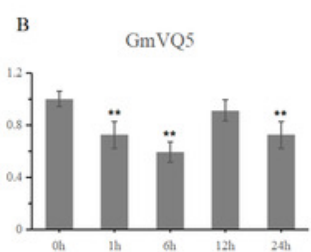

G GmVQ21
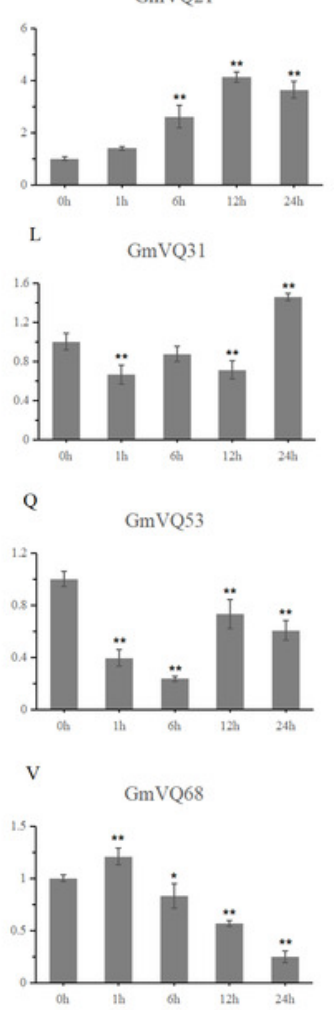

\section{Drought}
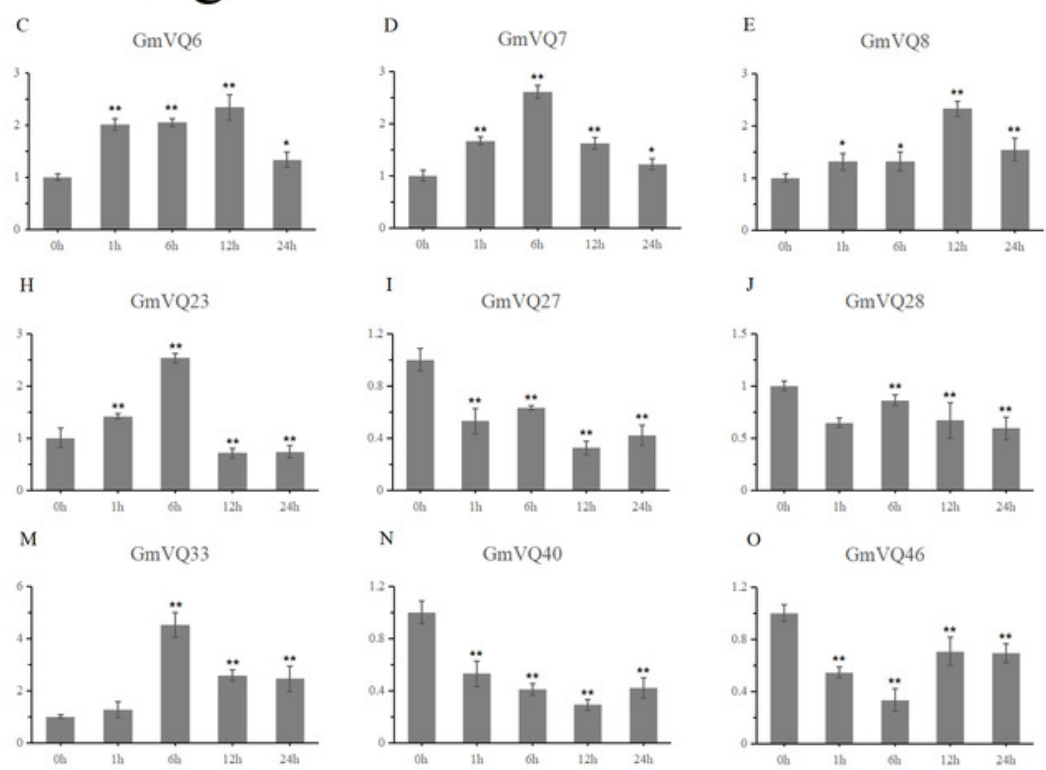

GmVQ28
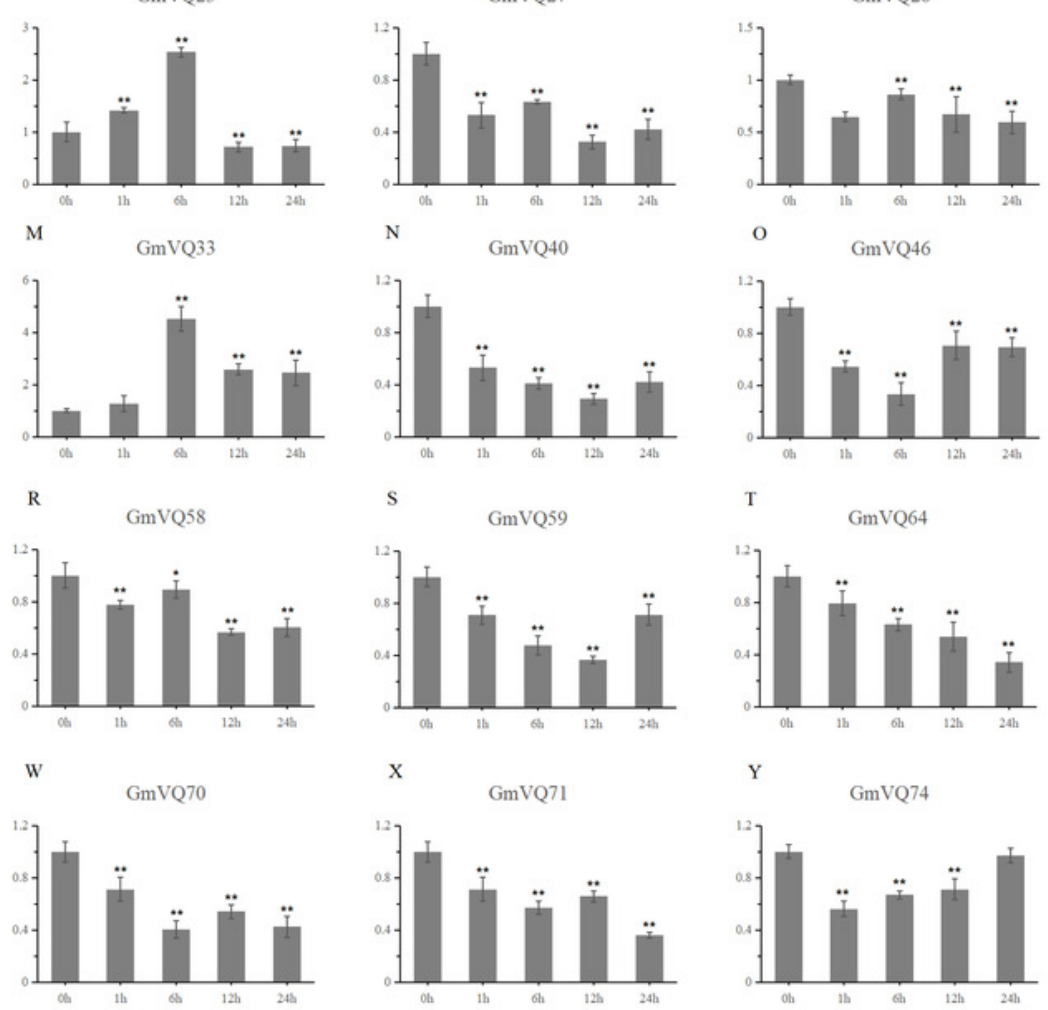

GmVQ74

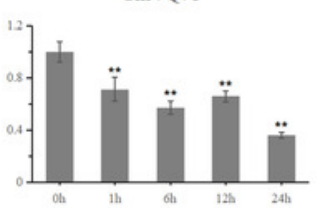

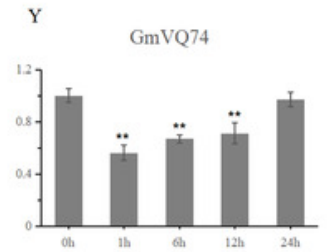


Figure 11

Number of each cis-acting element in the promoter region (1.5 kb upstream of the translation start site) of GmVQ genes. 


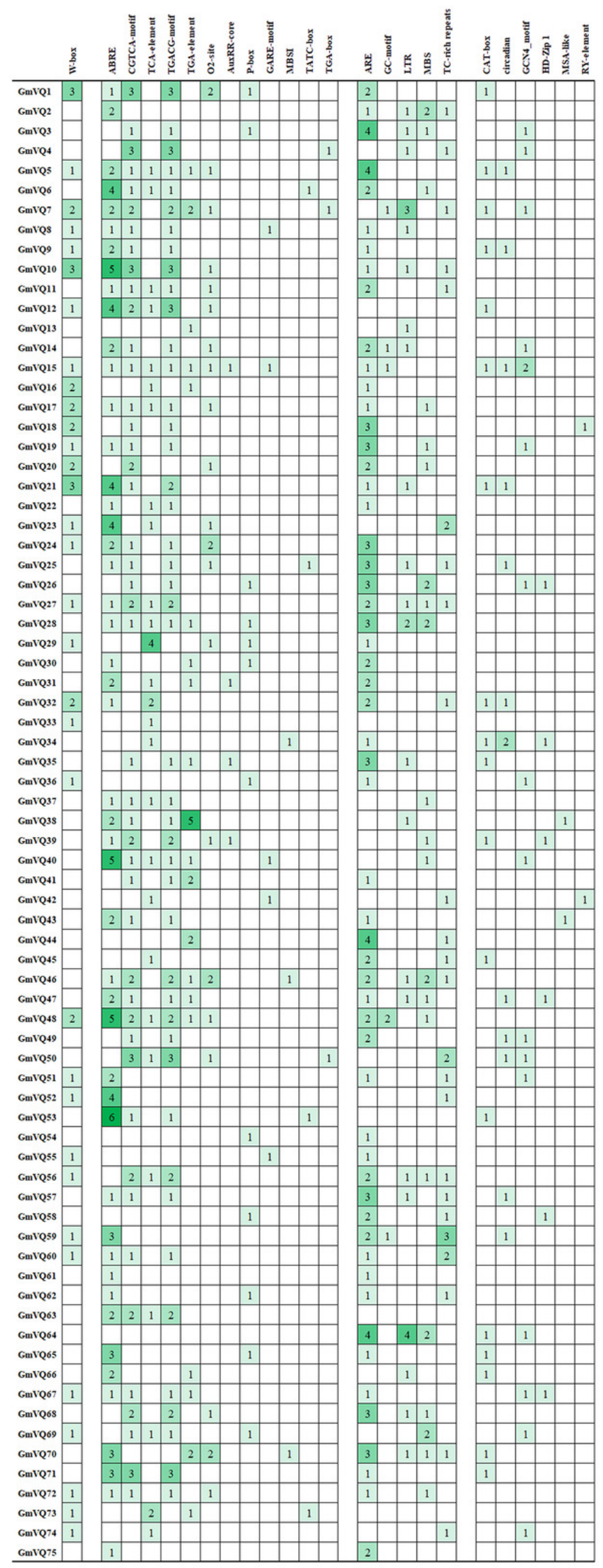




\section{Figure 12}

Interaction of GmVQ proteins with GmWRKY proteins.

(A) The prediction of interaction between GmVQ proteins and GmWRKY proteins by the PAIR website, and the interaction network was draw in Cytoscape 3.6.1. (B) Sequence analysis of the WRKY domains of GmWRKY proteins and AtWRKY proteins.

A

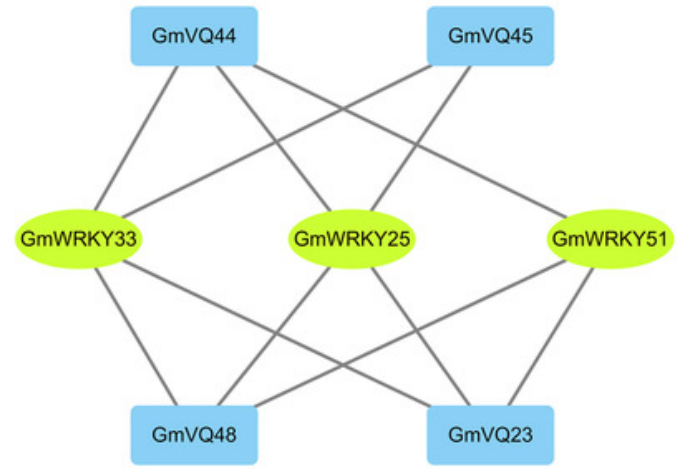

B

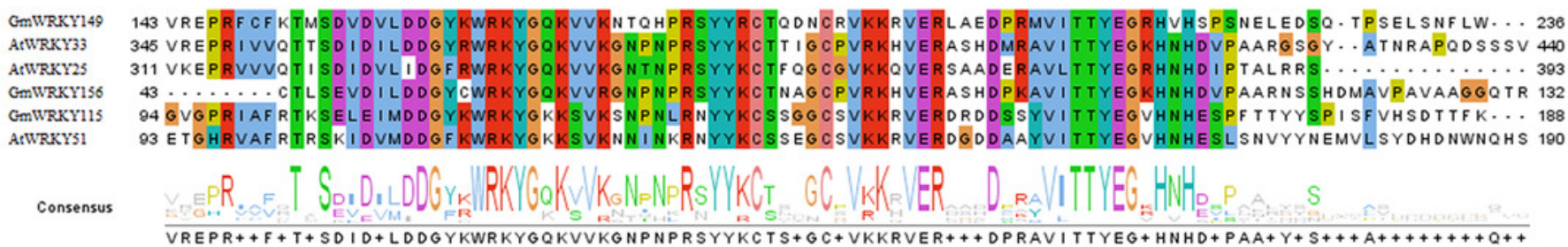




\section{Table 1 (on next page)}

Table 1 List of all GmVQ genes information identified in the Glycine max genome. 
Table 1 List of all GmVQ genes identified in the Glycine max genome

\begin{tabular}{|c|c|c|c|c|c|c|}
\hline Gene name & Gene locus & Chromosome location & Length (aa) & $\mathrm{pI}$ & Molecular weight (Da) & Family group \\
\hline GmVQ1 & Glyma01G018700 & chr1:1790049-1792039 & 318 & 10.66 & 34712.78 & VII \\
\hline GmVQ2 & Glyma01G096800 & $\operatorname{chr} 1: 31515839-31517715$ & 289 & 10.24 & 31636.98 & VII \\
\hline GmVQ3 & Glyma01G195300 & $\operatorname{chr} 1: 52952165-52953181$ & 154 & 9.48 & 16878.08 & VII \\
\hline GmVQ4 & Glyma02G208800 & chr2:39393500-39394691 & 212 & 9.96 & 23346.3 & VII \\
\hline GmVQ5 & Glyma03G120700 & chr3:33242128-33243660 & 233 & 7.79 & 24442.59 & VI \\
\hline GmVQ6 & Glyma03G127800 & chr3:34231323-34232268 & 167 & 4.79 & 18699.76 & I \\
\hline GmVQ7 & Glyma03G204900 & chr3:41299415-41300202 & 119 & 9.84 & 13358.41 & II \\
\hline GmVQ8 & Glyma03G249100 & chr3:44529232-44529956 & 127 & 9.11 & 14693.84 & I \\
\hline GmVQ9 & Glyma04G099600 & chr4:9115245-9116947 & 287 & 10.12 & 31433.71 & VII \\
\hline GmVQ10 & Glyma04G103200 & chr4:9567274-9568529 & 205 & 9.14 & 22493.86 & V \\
\hline GmVQ11 & Glyma04G103300 & chr4:9570059-9571000 & 313 & 6.64 & 34199.48 & V \\
\hline GmVQ12 & Glyma04G134200 & chr4:19214276-19215243 & 127 & 6.7 & 14519.25 & I \\
\hline GmVQ13 & Glyma04G214700 & chr4:48626650-48627708 & 212 & 5.9 & 22930.94 & II \\
\hline GmVQ14 & Glyma04G239400 & chr4:50786868-50788346 & 240 & 8.96 & 26255.85 & II \\
\hline GmVQ15 & Glyma05G107500 & chr5:28551166-28551996 & 186 & 8.48 & 20503.94 & VII \\
\hline GmVQ16 & Glyma05G133000 & chr5:32592583-32593521 & 211 & 7.79 & 23513.49 & V \\
\hline GmVQ17 & Glyma05G140700 & chr5:33359443-33360263 & 113 & 5.43 & 12313.85 & III \\
\hline GmVQ18 & Glyma05G179700 & chr5:36744975-36747458 & 486 & 6.12 & 52793.72 & V \\
\hline GmVQ19 & Glyma05G190000 & chr5:37570564-37571544 & 208 & 6.84 & 22399.46 & II \\
\hline GmVQ20 & Glyma05G198400 & chr5:38262465-38265127 & 186 & 9.52 & 20618.64 & IV \\
\hline GmVQ21 & Glyma06G101400 & chr6:8043143-8044571 & 295 & 10.28 & 32310.83 & VII \\
\hline GmVQ22 & Glyma06G104400 & chr6:8309457-8311289 & 341 & 6.06 & 37163.76 & $\mathrm{~V}$ \\
\hline GmVQ23 & Glyma06G104500 & chr6:8314638-8315685 & 316 & 6.48 & 34579.04 & V \\
\hline GmVQ24 & Glyma06G124400 & chr6:10128263-10129012 & 249 & 8.11 & 27136.69 & II \\
\hline GmVQ25 & Glyma06G151400 & chr6:12350255-12351217 & 222 & 5.97 & 24195.35 & II \\
\hline GmVQ26 & Glyma06G240300 & chr6:39620687-39622549 & 244 & 7.79 & 26839.89 & IV \\
\hline GmVQ27 & Glyma07G028700 & $\operatorname{chr} 7: 2307932-2309158$ & 193 & 9.98 & 20948.74 & IV \\
\hline GmVQ28 & Glyma07G092500 & chr7:8632559-8633302 & 247 & 5.97 & 27087.07 & II \\
\hline GmVQ29 & Glyma07G198000 & chr7:36647302-36650373 & 310 & 8.42 & 33741.87 & IV \\
\hline GmVQ30 & Glyma08G005700 & chr8:456890-461071 & 174 & 9.1 & 19216.73 & IV \\
\hline GmVQ31 & Glyma08G041900 & chr8:3320834-3322022 & 140 & 6.9 & 15589.54 & II \\
\hline GmVQ32 & Glyma08G087400 & chr8:6616587-6617687 & 221 & 6.91 & 24350.54 & V \\
\hline GmVQ33 & Glyma08G096000 & chr8:7331414-7331749 & 111 & 6.26 & 12060.59 & III \\
\hline GmVQ34 & Glyma08G137300 & chr8:10495440-10498069 & 472 & 6.33 & 51419.34 & V \\
\hline
\end{tabular}




\begin{tabular}{|c|c|c|c|c|c|}
\hline GmVQ35 & Glyma08G147600 & chr8:11258747-11259761 & 198 & 6.51 & 21201.12 \\
\hline GmVQ36 & Glyma08G157900 & chr8:12235183-12236400 & 141 & 5.63 & 15964.98 \\
\hline GmVQ37 & Glyma08G176500 & chr8:14151104-14151373 & 89 & 7.89 & 10029.37 \\
\hline GmVQ38 & Glyma08G214100 & chr8:17287863-17288952 & 194 & 9.69 & 21099.11 \\
\hline GmVQ39 & Glyma08G272000 & chr8:35627645-35629488 & 292 & 10.39 & 32000.2 \\
\hline GmVQ40 & Glyma08G272100 & chr8:35632723-35638206 & 361 & 9.8 & 39876.98 \\
\hline GmVQ41 & Glyma08G272200 & chr8:35665488-35667249 & 299 & 10.24 & 32844.1 \\
\hline GmVQ42 & Glyma08G308400 & chr8:42711855-42712403 & 182 & 4.3 & 20461.87 \\
\hline GmVQ43 & Glyma09G051900 & chr9:4508892-4509626 & 244 & 6.48 & 27252.83 \\
\hline GmVQ44 & Glyma09G111800 & chr9:22128197-22129301 & 203 & 7.11 & 22686.53 \\
\hline GmVQ45 & Glyma09G183700 & chr9:40881519-40882250 & 243 & 6.13 & 26618.56 \\
\hline GmVQ46 & Glyma10G273300 & chr10:49575568-49576678 & 191 & 7.83 & 20981.58 \\
\hline GmVQ47 & Glyma11G046400 & chr11:3468797-3469599 & 155 & 9.16 & 16952.31 \\
\hline GmVQ48 & Glyma11G239600 & chr11:33399330-33401730 & 439 & 7.02 & 47710.64 \\
\hline GmVQ49 & Glyma12G153600 & chr12:23455875-23457485 & 248 & 7.02 & 27499.55 \\
\hline GmVQ50 & Glyma12G225200 & chr12:38479959-38482769 & 246 & 7.17 & 27184.17 \\
\hline GmVQ51 & Glyma13G005100 & chr13:1422443-1424046 & 224 & 10.74 & 23979.57 \\
\hline GmVQ52 & Glyma13G039800 & chr13:12310527-12311616 & 240 & 10.11 & 25791.06 \\
\hline GmVQ53 & Glyma13G178500 & chr13:29211898-29216138 & 281 & 9.8 & 30474.04 \\
\hline GmVQ54 & Glyma13G193800 & chr13:30709903-30710253 & 116 & 5.14 & 13478 \\
\hline GmVQ55 & Glyma13G218400 & chr13:33181476-33181778 & 100 & 9.05 & 11045.26 \\
\hline GmVQ56 & Glyma13G238100 & chr13:34835450-34840141 & 260 & 9.54 & 27989.72 \\
\hline GmVQ57 & Glyma13G276100 & chr13:37756820-37759545 & 249 & 7.91 & 27358.41 \\
\hline GmVQ58 & Glyma14G002800 & $\operatorname{chr14:293552-294736}$ & 161 & 9.68 & 17490.39 \\
\hline GmVQ59 & Glyma14G124800 & chr14:19432507-19433220 & 237 & 8.85 & 25366.52 \\
\hline GmVQ60 & Glyma14G172200 & chr14:42617341-42619795 & 429 & 6.59 & 46407.55 \\
\hline GmVQ61 & Glyma15G075200 & chr15:5769826-5772617 & 199 & 9.77 & 21595.31 \\
\hline GmVQ62 & Glyma15G158200 & chr15:13251793-13252987 & 252 & 7.16 & 27774.66 \\
\hline GmVQ63 & Glyma15G232200 & chr15:43662201-43662912 & 122 & 6.65 & 14310.95 \\
\hline GmVQ64 & Glyma15G249800 & chr15:47637825-47638440 & 89 & 7.89 & 10074.51 \\
\hline GmVQ65 & Glyma15G268300 & chr15:50482677-50484349 & 158 & 7.74 & 17892.46 \\
\hline GmVQ66 & Glyma17G159600 & chr17:13790434-13791675 & 190 & 9.3 & 21149.77 \\
\hline GmVQ67 & Glyma17G182600 & $\operatorname{chr17:22616386-22616835}$ & 149 & 9.27 & 16873.05 \\
\hline GmVQ68 & Glyma18G017800 & $\operatorname{chr} 18: 1285879-1287658$ & 454 & 6.45 & 48264.96 \\
\hline GmVQ69 & Glyma18G108600 & chr18:12426789-12427328 & 179 & 4.29 & 20004.4 \\
\hline GmVQ70 & Glyma19G125300 & chr19:38346007-38347425 & 232 & 9.64 & 24435.6 \\
\hline GmVQ71 & Glyma19G130400 & chr19:39031134-39032107 & 168 & 5.16 & 18878.02 \\
\hline
\end{tabular}




\begin{tabular}{lllcccc} 
GmVQ72 & Glyma19G202300 & chr19:45923283-45923992 & 124 & 9.7 & 13533.53 & II \\
GmVQ73 & Glyma19G246700 & chr19:49331581-49332332 & 102 & 9.19 & 11775.23 & I \\
GmVQ74 & Glyma20G064500 & chr20:21930212-21930913 & 233 & 10.51 & 25070.57 & V \\
GmVQ75 & Glyma20G116600 & chr20:35927408-35928282 & 157 & 5.9 & 17333.32 & II \\
\hline
\end{tabular}

1 


\section{Table 2 (on next page)}

Table 2 List of paralogous and orthologous pairs between soybean and Arabidopsis thaliana. 
Table 2 Paralogous (Gm-Gm) and orthologous (Gm-At) gene pairs

\begin{tabular}{|c|c|c|}
\hline Gm-Gm & Gm-Gm & $\mathrm{Gm}-\mathrm{At}$ \\
\hline GmVQ3/GmVQ47 & GmVQ24/GmVQ59 & GmVQ37/AtVQ1 \\
\hline GmVQ5/GmVQ70 & GmVQ27/GmVQ38 & GmVQ64/AtVQ1 \\
\hline GmVQ6/GmVQ71 & GmVQ28/GmVQ45 & GmVQ14/AtVQ3 \\
\hline GmVQ7/GmVQ72 & GmVQ29/GmVQ53 & GmVQ24/AtVQ3 \\
\hline GmVQ8/GmVQ73 & GmVQ29/GmVQ61 & GmVQ52/AtVQ3 \\
\hline GmVQ9/GmVQ21 & GmVQ29/GmVQ56 & GmVQ59/AtVQ3 \\
\hline GmVQ10/GmVQ11 & GmVQ34/GmVQ68 & GmVQ29/AtVQ5 \\
\hline GmVQ10/GmVQ22 & GmVQ34/GmVQ48 & GmVQ53/AtVQ5 \\
\hline GmVQ10/GmVQ23 & GmVQ37/GmVQ64 & GmVQ61/AtVQ5 \\
\hline GmVQ10/GmVQ67 & GmVQ39/GmVQ40 & GmVQ56/AtVQ5 \\
\hline GmVQ11/GmVQ22 & GmVQ39/GmVQ41 & GmVQ46/AtVQ8 \\
\hline GmVQ11/GmVQ23 & GmVQ39/GmVQ2 & GmVQ75/AtVQ8 \\
\hline GmVQ11/GmVQ67 & GmVQ40/GmVQ41 & GmVQ9/AtVQ9 \\
\hline GmVQ13/GmVQ25 & GmVQ40/GmVQ2 & GmVQ21/AtVQ9 \\
\hline GmVQ14/GmVQ24 & GmVQ41/GmVQ2 & GmVQ37/AtVQ10 \\
\hline GmVQ14/GmVQ52 & GmVQ42/GmVQ69 & GmVQ64/AtVQ10 \\
\hline GmVQ14/GmVQ59 & GmVQ43/GmVQ62 & GmVQ27/AtVQ11 \\
\hline GmVQ15/GmVQ66 & GmVQ46/GmVQ75 & GmVQ38/AtVQ11 \\
\hline GmVQ16/GmVQ32 & GmVQ49/GmVQ26 & GmVQ1/AtVQ14 \\
\hline GmVQ18/GmVQ34 & GmVQ50/GmVQ57 & GmVQ5/AtVQ15 \\
\hline GmVQ18/GmVQ68 & GmVQ51/GmVQ74 & GmVQ70/AtVQ15 \\
\hline GmVQ18/GmVQ48 & GmVQ52/GmVQ59 & GmVQ44/AtVQ17 \\
\hline GmVQ19/GmVQ35 & GmVQ53/GmVQ61 & GmVQ50/AtVQ19 \\
\hline GmVQ20/GmVQ30 & GmVQ53/GmVQ56 & GmVQ57/AtVQ19 \\
\hline GmVQ22/GmVQ23 & GmVQ54/GmVQ63 & GmVQ28/AtVQ20 \\
\hline GmVQ22/GmVQ67 & GmVQ61/GmVQ56 & GmVQ45/AtVQ20 \\
\hline GmVQ23/GmVQ67 & GmVQ65/GmVQ36 & GmVQ19/AtVQ21 \\
\hline \multirow[t]{10}{*}{ GmVQ24/GmVQ52 } & GmVQ68/GmVQ48 & GmVQ35/AtVQ21 \\
\hline & & GmVQ5/AtVQ24 \\
\hline & & GmVQ70/AtVQ24 \\
\hline & & GmVQ44/AtVQ25 \\
\hline & & GmVQ20/AtVQ31 \\
\hline & & GmVQ30/AtVQ31 \\
\hline & & GmVQ18/AtVQ34 \\
\hline & & GmVQ34/AtVQ34 \\
\hline & & GmVQ68/AtVQ34 \\
\hline & & GmVQ48/AtVQ34 \\
\hline
\end{tabular}




\section{Table 3 (on next page)}

Table 3 List of Ka, Ks and Ka/Ks values calculated for paralogous VQ gene pairs. 
Table $3 \mathrm{Ka}$, Ks and Ka/Ks values calculated for paralogous VQ gene pairs

\begin{tabular}{|c|c|c|c|c|}
\hline Gene 1 & Gene 2 & $\mathrm{Ka}$ & $\mathrm{Ks}$ & $\mathrm{Ka} / \mathrm{Ks}$ ratio \\
\hline GmVQ10 & GmVQ11 & 0.002146692 & 0.013590406 & 0.15795643 \\
\hline GmVQ39 & GmVQ40 & 0.014329244 & 0.032130119 & 0.445975451 \\
\hline GmVQ54 & GmVQ63 & 0.070550548 & 0.066958938 & 1.053638991 \\
\hline GmVQ65 & GmVQ36 & 0.129398947 & 0.090241867 & 1.433912564 \\
\hline GmVQ39 & GmVQ41 & 0.015091052 & 0.092583143 & 0.163 \\
\hline GmVQ37 & GmVQ64 & 0.033904078 & 0.095366382 & 0.355513935 \\
\hline GmVQ40 & GmVQ41 & 0.02957328 & 0.096785754 & 0.305554062 \\
\hline GmVQ42 & GmVQ69 & 0.039057024 & 0.110714815 & 0.352771436 \\
\hline GmVQ16 & GmVQ32 & 0.056833182 & 0.117015316 & 0.485690114 \\
\hline GmVQ40 & GmVQ2 & 0.056768509 & 0.119061292 & 0.476800713 \\
\hline GmVQ50 & GmVQ57 & 0.012662345 & 0.127110953 & 0.099616473 \\
\hline GmVQ49 & GmVQ26 & 0.034291687 & 0.128532842 & 0.26679319 \\
\hline GmVQ43 & GmVQ62 & 0.057675771 & 0.129647567 & 0.444865819 \\
\hline GmVQ19 & GmVQ35 & 0.060650867 & 0.132943691 & 0.456214704 \\
\hline GmVQ20 & GmVQ30 & 0.047369299 & 0.134768712 & 0.351485879 \\
\hline GmVQ7 & GmVQ72 & 0.073853375 & 0.1352768 & 0.545942653 \\
\hline GmVQ39 & GmVQ2 & 0.050072412 & 0.136741168 & 0.366183886 \\
\hline GmVQ18 & GmVQ34 & 0.048240383 & 0.141323284 & 0.341347734 \\
\hline GmVQ68 & GmVQ48 & 0.072358564 & 0.146626964 & 0.493487433 \\
\hline GmVQ3 & GmVQ47 & 0.082715722 & 0.153202253 & 0.539911912 \\
\hline GmVQ5 & GmVQ70 & 0.053173881 & 0.15994977 & 0.33244112 \\
\hline GmVQ46 & GmVQ75 & 0.053993084 & 0.162730183 & 0.33179514 \\
\hline GmVQ41 & GmVQ2 & 0.047558234 & 0.164392307 & 0.289297198 \\
\hline GmVQ10 & GmVQ22 & 0.080947528 & 0.167701359 & 0.482688565 \\
\hline GmVQ22 & GmVQ23 & 0.12358775 & 0.173118789 & 0.713889871 \\
\hline GmVQ51 & GmVQ74 & 0.051867494 & 0.17525146 & 0.295960409 \\
\hline GmVQ27 & GmVQ38 & 0.068641212 & 0.187083237 & 0.366901992 \\
\hline GmVQ8 & GmVQ73 & 0.07651417 & 0.194357737 & 0.393676994 \\
\hline GmVQ15 & GmVQ66 & 0.064674193 & 0.201500105 & 0.32096357 \\
\hline GmVQ9 & GmVQ21 & 0.031810279 & 0.204712416 & 0.155390081 \\
\hline GmVQ11 & GmVQ22 & 0.068482759 & 0.20561255 & 0.33306702 \\
\hline GmVQ28 & GmVQ45 & 0.07428748 & 0.212707642 & 0.349246879 \\
\hline GmVQ13 & GmVQ25 & 0.0495425 & 0.21323249 & 0.232340297 \\
\hline GmVQ61 & GmVQ56 & 0.078572076 & 0.228081862 & 0.344490682 \\
\hline GmVQ11 & GmVQ67 & 0.187484416 & 0.242381253 & 0.773510383 \\
\hline GmVQ14 & GmVQ24 & 0.070535521 & 0.260458812 & 0.270812571 \\
\hline GmVQ11 & GmVQ23 & 0.143077885 & 0.278054006 & 0.514568687 \\
\hline GmVQ10 & GmVQ67 & 0.25396485 & 0.28526939 & 0.890263236 \\
\hline GmVQ6 & GmVQ71 & 0.098963625 & 0.304675089 & 0.324816923 \\
\hline GmVQ22 & GmVQ67 & 0.242648421 & 0.35967981 & 0.674623413 \\
\hline GmVQ29 & GmVQ53 & 0.1121903 & 0.365440906 & 0.306999841 \\
\hline GmVQ10 & GmVQ23 & 0.206060196 & 0.446610645 & 0.461386664 \\
\hline GmVQ18 & GmVQ48 & 0.431966031 & 0.855432366 & 0.50496807 \\
\hline GmVQ18 & GmVQ68 & 0.415845352 & 0.884745464 & 0.470016936 \\
\hline GmVQ34 & GmVQ68 & 0.40356234 & 0.937434864 & 0.430496406 \\
\hline GmVQ34 & GmVQ48 & 0.385776641 & 0.968352827 & 0.398384381 \\
\hline GmVQ29 & GmVQ61 & 0.320802002 & 0.987438675 & 0.324882963 \\
\hline GmVQ24 & GmVQ59 & 0.231784771 & 1.001714144 & 0.231388139 \\
\hline GmVQ14 & GmVQ59 & 0.271393455 & 1.079747859 & 0.251348917 \\
\hline GmVQ23 & GmVQ67 & 0.564635744 & 1.087320132 & 0.51929117 \\
\hline GmVQ53 & GmVQ61 & 0.281138217 & 1.090000891 & 0.257924759 \\
\hline GmVQ29 & GmVQ56 & 0.422850584 & 1.281581327 & 0.329944401 \\
\hline GmVQ53 & GmVQ56 & 0.280639233 & 1.337494009 & 0.209824665 \\
\hline GmVQ14 & GmVQ52 & 1.027115432 & 1.538918692 & 0.667426705 \\
\hline GmVQ24 & GmVQ52 & 1.074280372 & 1.754750076 & 0.612212751 \\
\hline
\end{tabular}

1 